TRANSACTIONS OF THE

AMERICAN MATHEMATICAL SOCIETY

Volume 361, Number 2, February 2009, Pages 951-977

S 0002-9947(08)04584-4

Article electronically published on August 19, 2008

\title{
FAST AND STRONGLY LOCALIZED OBSERVATION FOR THE SCHRÖDINGER EQUATION
}

\author{
G. TENENBAUM AND M. TUCSNAK
}

\begin{abstract}
We study the exact observability of systems governed by the Schrödinger equation in a rectangle with homogeneous Dirichlet (respectively Neumann) boundary conditions and with Neumann (respectively Dirichlet) boundary observation. Generalizing results from Ramdani, Takahashi, Tenenbaum and Tucsnak (2005), we prove that these systems are exactly observable in in arbitrarily small time. Moreover, we show that the above results hold even if the observation regions have arbitrarily small measures. More precisely, we prove that in the case of homogeneous Neumann boundary conditions with Dirichlet boundary observation, the exact observability property holds for every observation region with nonempty interior. In the case of homogeneous Dirichlet boundary conditions with Neumann boundary observation, we show that the exact observability property holds if and only if the observation region has an open intersection with an edge of each direction. Moreover, we give explicit estimates for the blow-up rate of the observability constants as the time and (or) the size of the observation region tend to zero. The main ingredients of the proofs are an effective version of a theorem of Beurling and Kahane on nonharmonic Fourier series and an estimate for the number of lattice points in the neighbourhood of an ellipse.
\end{abstract}

\section{Introduction AND MAIN RESUlts}

The exact observability and its dual property, the exact controllability, of systems governed by Schrödinger equations have been extensively studied; see, for instance, Jaffard [15, Lebeau [18, Burq and Zworski [6], Komornik [17] and the references therein. The observation operators that have been considered are either distributed in the domain (internal observation) or localized at the boundary (boundary observation).

It is usually assumed, in the existing literature, that the observation region satisfies the geometric optics condition of Bardos, Lebeau and Rauch [3, which is known to be necessary and sufficient for the exact observability of the wave equation. In the case of internal control, the first result asserting that exact observability for the Schrödinger equation holds for an arbitrarily small control region has been given by Jaffard [15], who shows, in particular, that for systems governed by the Schrödinger equation in a rectangle we have exact internal observability with an arbitrary observation region and in arbitrarily small time. However, Jaffard's method does not yield an estimate on the constant in the observability inequality.

Received by the editors April 20, 2007.

2000 Mathematics Subject Classification. Primary 93C25, 93B07, 93C20, 11N36.

Key words and phrases. Boundary exact observability, Schrödinger equation, plate equation, sieve, quadratic forms, squares.

(C)2008 American Mathematical Society 
Other observability results violating the geometric condition of Bardos, Lebeau and Rauch have been obtained in [6] for partially rectangular domains, such as the Bunimovich stadium and the square with a hole. However, the exact internal observability with an arbitrarily small observation region cannot be generalized for an arbitrary domain: see, for instance, Chen, Fulling, Narcowich and Sun [7, where it is shown that, for the Schrödinger equation in a disk, the exact internal observability property fails if the observation region does not touch the boundary.

The first result establishing exact boundary observability for the Schrödinger equation with an arbitrarily small observation region has been given by Ramdani, Takahashi, Tenenbaum and Tucsnak [22, where the observed quantity is the Dirichlet or the Neumann boundary trace of the solution.

The present work is devoted to obtaining new information in this direction:

- We prove new, exact boundary observability results improving those in 22 in two directions: we are able to replace square domains by rectangles and we show that the conclusion holds even for an arbitrarily small observation time.

- We provide, in some cases, explicit estimates for the observability constants in terms of the observability time and of the size of the observation region. To our knowledge, these are the first estimates of such type for the Schrödinger equation in several space dimensions and with arbitrarily small observation regions. We refer to Miller [20] and to Tenenbaum and Tucsnak [26] for the corresponding estimates with "large" observation regions.

From a qualitative point of view, the above described results essentially amount to the statement (see Theorem 4.2 below) that, for any given $u, v \in] 0, \infty[$ and any nonempty open set $\mathscr{U} \subset \mathbb{R}^{2}$, there exists $\delta=\delta(\mathscr{U})=\delta(\mathscr{U} ; u, v)>0$ such that

$$
\int_{\mathscr{U}}\left|\sum_{m, n \in \mathbb{Z}} a_{m n} \mathrm{e}^{2 \pi i\left\{n x+\left(u m^{2}+v n^{2}\right) t\right\}}\right|^{2} \mathrm{~d} x \mathrm{~d} t \geqslant \delta(\mathscr{U}) \sum_{m, n \in \mathbb{Z}}\left|a_{m n}\right|^{2}
$$

for all sequences $\left(a_{m n}\right) \in \ell^{2}(\mathbb{Z} \times \mathbb{Z}, \mathbb{C})$. This, in turn, is shown by deriving an effective version of an inequality of Beurling and Kahane and by obtaining quantitative estimates for the number of lattice points in the neighbourhood of an ellipse. The latter are obtained via techniques from analytic number theory.

In order to state our results precisely, we denote by $\Omega$ the rectangle $] 0, a[\times] 0, b[$, with $a, b>0$, and we consider the following initial and boundary value problem (of unknown $w=w(x, t)$, with $x \in \Omega$ and $t \geqslant 0$ ):

$$
\begin{cases}\dot{w}+i \Delta w=0 & (x \in \Omega, t \geqslant 0), \\ \frac{\partial w}{\partial \nu}=0 & (x \in \partial \Omega, t \geqslant 0), \\ w(x, 0)=\psi(x) & (x \in \Omega) .\end{cases}
$$

Here and in the sequel, a dot denotes differentiation with respect to the time $t$ and $\frac{\partial}{\partial \nu}$ stands for the normal derivative operator. We use the standard notation $H^{m}(\Omega)$ $(m \in \mathbb{Z})$ and $H_{0}^{m}(\Omega)(m \in \mathbb{N})$ for the Sobolev spaces on $\Omega$.

We can now state our first main result. 
Theorem 1.1. Let $\Gamma$ be a nonempty open subset of $\partial \Omega$, let $T>0$ and let $w=w(\psi)$ be the solution of (1.1). Then

$$
C_{T, \Gamma}:=\sup _{\psi \in H_{0}^{1}(\Omega), \psi \neq \equiv 0} \frac{\|\psi\|_{L^{2}(\Omega)}}{\|w\|_{L^{2}\left([0, T] ; L^{2}(\Gamma)\right)}}<\infty .
$$

Moreover, if $a / b \in \mathbb{Q}$ and $\Gamma \supset I_{1} \times\{0\}$, where $I_{1}$ is a subinterval of $[0, a]$ with positive length $\left|I_{1}\right|$, then there exist constants $K_{1}, K_{2}$, depending only on a and $b$, such that

$$
C_{T, \Gamma} \leqslant \exp \left\{K_{1} \frac{\left(\ln \left|I_{1}\right|\right)^{2}}{\left|I_{1}\right|}+\mathrm{e}^{K_{2} / T^{2}}\right\} \quad(T>0) .
$$

Remark 1.2. In control theoretic terms, the above theorem asserts that the observation system, with state space $L^{2}(\Omega)$ and output space $L^{2}(\Gamma)$, determined by (1.1) and the output law $y=\left.w\right|_{\Gamma}$ is exactly observable in any time $T>0$. In order to give a functional analytic interpretation of (1.3) we introduce, for each $T>0$, the map $\psi \mapsto G_{T} \psi$ defined by

$$
\left(G_{T} \psi\right)(t)=\left.w(\cdot, t)\right|_{\Gamma} \quad(t \in[0, T]),
$$

where $w$ is the solution of (1.1). It is not difficult to check that $G_{T}$ is a bounded linear operator from $L^{2}(\Omega)$ to $L^{2}\left([0, T] ; L^{2}(\Gamma)\right)$. By the closed graph theorem, condition (1.2) implies that the set $\mathcal{H}_{T}$ of the operators $H \in \mathcal{L}\left(L^{2}\left([0, T] ; L^{2}(\Gamma)\right), L^{2}(\Omega)\right)$ such that $H G_{T}=I$ is nonempty and has a unique minimal element $H_{T}$, in the sense that

$$
H_{T} H_{T}^{*} \leqslant H H^{*} \quad\left(H \in \mathcal{H}_{T}\right) .
$$

It is easy to check that

$$
\left\|H_{T}\right\|_{\mathcal{L}\left(L^{2}\left([0, T] ; L^{2}(\Gamma)\right), L^{2}(\Omega)\right)}=C_{T, \Gamma}
$$

so that the norm of $H_{T}$ is bounded by the right-hand side of (1.3).

By a standard duality argument, Theorem 1.1 implies the following exact controllability result and control cost estimate. We refer to 26] for the precise definition of these concepts.

Corollary 1.3. For any nonempty open subset $\Gamma$ of $\partial \Omega$, the system

$$
\begin{cases}\dot{w}-i \Delta w=0 & (x \in \Omega, t \geqslant 0), \\ w=0 & (x \in \partial \Omega \backslash \Gamma, t \geqslant 0), \\ w=u \in L^{2}\left([0, T] ; L^{2}(\Gamma)\right) & (x \in \Gamma, t \geqslant 0), \\ w(x, 0)=\psi(x) & (x \in \Omega),\end{cases}
$$

with the control function $u$, is exactly controllable in any time $T>0$ in the state space $L^{2}(\Omega)$. Moreover, the control cost in time $T$ and with support $\Gamma$ coincides with the constant $C_{T, \Gamma}$ defined in (1.2).

In the case of the Schrödinger equation with Dirichlet boundary conditions and with Neumann observation the situation is slightly different. The exact observability in the "natural" space $H_{0}^{1}(\Omega)$ holds only if a simple geometric condition is satisfied. In order to give the precise statement of this result, we denote respectively by

$$
\begin{aligned}
& \Gamma_{1}:=([0, a] \times\{0\}) \cup([0, a] \times\{b\}), \\
& \Gamma_{2}:=(\{0\} \times[0, b]) \cup(\{a\} \times[0, b]),
\end{aligned}
$$


the horizontal and vertical parts of $\partial \Omega$ and we consider the initial and boundary value problem:

$$
\begin{cases}\dot{w}+i \Delta w=0 & (x \in \Omega, t \geqslant 0) \\ w=0 & (x \in \partial \Omega, t \geqslant 0) \\ w(x, 0)=\psi(x) & (x \in \Omega) .\end{cases}
$$

Theorem 1.4. Let $\Gamma$ be an open subset of $\partial \Omega$, let $T>0$ and let $w=w(\psi)$ be the solution of (1.4). Then, the following statements are equivalent:

(S1): The region $\Gamma$ contains both a horizontal and a vertical segment of nonzero length, i.e. $\Gamma \cap \Gamma_{i} \neq \varnothing$ for $i \in\{1,2\}$.

(S2): We have

$$
Q_{T, \Gamma}:=\sup _{\substack{\psi \in H^{2}(\Omega) \cap H_{0}^{1}(\Omega) \\ \psi \neq 0}} \frac{\|\psi\|_{H_{0}^{1}(\Omega)}}{\left\|\frac{\partial w}{\partial \nu}\right\|_{L^{2}\left([0, T] ; L^{2}(\Gamma)\right)}}<\infty .
$$

Moreover, if $a / b \in \mathbb{Q}$, if condition $(S 1)$ is satisfied and if

$$
\Gamma \supset\left(I_{1} \times\{0\}\right) \cup\left(\{0\} \times I_{2}\right),
$$

where $I_{1}, I_{2}$ are respectively subintervals of $[0, a]$ and $[0, b]$ with positive lengths $\left|I_{1}\right|$, $\left|I_{2}\right|$, then, there exist constants $K_{1}, K_{2}, K_{3}$, depending only on $a$ and $b$, such that

$$
Q_{T, \Gamma} \leqslant \exp \left\{K_{1} \frac{\left(\ln \left|I_{1}\right|\right)^{2}}{\left|I_{1}\right|}+K_{2} \frac{\left(\ln \left|I_{2}\right|\right)^{2}}{\left|I_{2}\right|}+\mathrm{e}^{K_{3} / T^{2}}\right\} .
$$

Note that, as in Remark 1.2 inequality (1.6) can be interpreted in terms of an upper bound for the norm of an appropriate operator.

By duality, Theorem 1.4 implies the following exact controllability result and control cost estimate. With the notation in Proposition (1.4), consider the system

$$
\begin{cases}\dot{w}-i \Delta w=0 & (x \in \Omega, t>0), \\ w=0 & (x \in \partial \Omega \Gamma, t>0), \\ w=u \in L^{2}\left([0, T] ; L^{2}(\Gamma)\right) & (x \in \Gamma, t>0), \\ w(x, 0)=\psi(x) & (x \in \Omega) .\end{cases}
$$

Corollary 1.5. The following conditions are equivalent:

- The system (1.7) is exactly controllable in any time $T>0$ in the state space $X=H^{-1}(\Omega)$.

- We have $\Gamma \cap \Gamma_{i} \neq \varnothing$, for $i \in\{1,2\}$.

Moreover, the control cost in time $T$ and with support $\Gamma$ coincides with the constant $Q_{T, \Gamma}$ defined in (1.5).

Remark 1.6. It is well known (see, for instance, [18]) that the exact observability results for the Schrödinger equation yield observability estimates for the EulerBernoulli plate equation. We refer to [22] for precise forms of the boundary conditions and of the corresponding observation operators.

The remaining part of this paper is organized as follows. In Section 2 we give some notation and we introduce several notions and results used later. Section 3 is devoted to an effective version of an inequality of Kahane and Beurling. In Section 4 we prove our main results, appealing, in particular, to two new estimates of an arithmetical nature. These are proved in the last two sections. 


\section{NotATION AND PRELIMINARIES}

In this section, we introduce several functions used in Section 3 for the proof of Beurling-Kahane type inequalities and we recall some of their properties.

We start with some notation. Let $\mathrm{e}: \mathbb{R} \rightarrow \mathbb{C}$ be defined by $\mathrm{e}(t):=\mathrm{e}^{2 \pi i t}$ for all real $t$. We define the Fourier transform $\widehat{f}$ of a function $f \in L^{1}(\mathbb{R})$ by

$$
\widehat{f}(\xi)=\int_{\mathbb{R}} f(t) \mathrm{e}(-\xi t) \mathrm{d} t \quad(\xi \in \mathbb{R}) .
$$

We denote by $\operatorname{sgn}: \mathbb{R} \rightarrow\{0, \pm 1\}$ the usual sign function, defined for real $x$ by

$$
\operatorname{sgn}(x):= \begin{cases}1 & \text { if } x>0 \\ 0 & \text { if } x=0 \\ -1 & \text { if } x<0\end{cases}
$$

and we write, traditionally, $x^{+}:=\max \{x, 0\},\lfloor x\rfloor:=\max \{n \in \mathbb{Z}: n \leqslant x\}$ for $x \in \mathbb{R}$. For $A \subset \mathbb{R}$, we write $\mathbb{1}_{A}$ for the characteristic function of the set $A$.

We let $\ln _{k}$ designate the $k$-fold iterated natural logarithm.

In the sequel, we freely use, according to notational convenience, Landau's $O$ symbol or Vinogradov's «-notation. Thus $f(x) \ll g(x)(x \in X)$ indicates that, for all $x$ in the set $X$, the inequality $|f(x)| \leqslant C|g(x)|$ holds with a suitable constant $C>0$, which may depend on certain implicit parameters. In this last case, the dependence may be indicated by annotating the Vinogradov symbol with appropriate subscripts.

We write $f \asymp g$ to indicate that both estimates $f \ll g$ and $g \ll f$ hold simultaneously.

Let $d \in \mathbb{N}^{*}$. Following Kahane's terminology in [16], we say that a sequence $\Lambda=\left(\lambda_{n}\right)_{n \in \mathbb{Z}}$ in $\mathbb{R}^{d}$ is regular if

$$
\inf _{\substack{m, n \in \mathbb{Z} \\ m \neq n}}\left\|\lambda_{m}-\lambda_{n}\right\|>0
$$

where $\|\cdot\|$ stands for the euclidean norm in $\mathbb{R}^{d}$. Given a regular sequence $\Lambda$ in $\mathbb{R}^{d}$ and a sequence $\left(a_{n}\right) \in \ell^{2}(\mathbb{Z}, \mathbb{C})$, we may define an almost periodic function $f: \mathbb{R}^{d} \rightarrow \mathbb{C}$ by the almost everywhere convergent series

$$
f(x)=\sum_{n \in \mathbb{Z}} a_{n} \mathrm{e}\left(\lambda_{n} \cdot x\right) \quad\left(x \in \mathbb{R}^{d}\right),
$$

where $x \cdot y$ stands for the inner product of $x, y \in \mathbb{R}^{d}$.

Definition 2.1. Let $d \in \mathbb{N}$ and let $\Lambda$ be a regular sequence in $\mathbb{R}^{d}$. An open set $\mathscr{U} \subset \mathbb{R}^{d}$ is called a domain associated to (the sequence) $\Lambda$ if there exists a constant $\delta=\delta(\mathscr{U})>0$, such that

$$
\int_{\mathscr{U}}|f(x)|^{2} \mathrm{~d} x \geqslant \delta(\mathscr{U}) \sum_{n \in \mathbb{Z}}\left|a_{n}\right|^{2} \quad \forall\left(a_{n}\right) \in \ell^{2}(\mathbb{Z}, \mathbb{C}),
$$

where $f$ is defined by (2.1). 
We will need the following structural theorem of Kahane [16, Proposition III.3.1].

Proposition 2.2. Let $\Lambda_{1}, \Lambda_{2}$ be two regular sequences in $\mathbb{R}^{d}$, with $d \in \mathbb{N}^{*}$. Assume that $\mathscr{U}_{1} \subset \mathbb{R}^{d}$ (respectively, $\mathscr{U}_{2} \subset \mathbb{R}^{d}$ ) is a domain associated to $\Lambda_{1}$ (respectively, to $\Lambda_{2}$ ) and that the sequence $\Lambda=\Lambda_{1} \cup \Lambda_{2}$ is regular. Then any open set $\mathscr{U} \subset \mathbb{R}^{d}$ containing $\mathscr{U}_{1}+\mathscr{U}_{2}$ is a domain associated to $\Lambda$.

Consider the complex functions

$$
\begin{aligned}
& H(z):=\left(\frac{\sin \pi z}{\pi}\right)^{2}\left\{\sum_{m \in \mathbb{Z}} \frac{\operatorname{sgn}(m)}{(z-m)^{2}}+\frac{2}{z}\right\}, \\
& K(z)=\left(\frac{\sin \pi z}{\pi z}\right)^{2}, \\
& B(z):=K(z)+H(z), \quad b(z):=H(z)-K(z) .
\end{aligned}
$$

According to Beurling [4, we have

$$
\begin{gathered}
b(x) \leqslant \operatorname{sgn}(x) \leqslant B(x) \quad(x \in \mathbb{R}), \\
\int_{\mathbb{R}}\{B(x)-\operatorname{sgn}(x)\} \mathrm{d} x=\int_{\mathbb{R}}\{\operatorname{sgn}(x)-b(x)\} \mathrm{d} x=1 .
\end{gathered}
$$

Given a parameter $T>0$, we define two functions $m_{T}^{-}, m_{T}^{+}: \mathbb{R} \rightarrow \mathbb{R}$ by

$$
\left\{\begin{array}{l}
m_{T}^{-}(x) \quad:=\frac{1}{2}\{b(T+x)+b(T-x)\} \\
m_{T}^{+}(x) \quad:=\frac{1}{2}\{B(T+x)+B(T-x)\}
\end{array} \quad(x \in \mathbb{R}) .\right.
$$

Proposition 2.3. We have

$$
m_{T}^{-}(x) \leqslant \mathbb{1}_{[-T, T]}(x) \leqslant m_{T}^{+}(x) \quad(x \in \mathbb{R}),
$$

and

$$
\widehat{m_{T}^{ \pm}}(\xi)= \begin{cases}\frac{\sin (2 \pi T|\xi|)}{\pi}+(1-|\xi|) \frac{\sin \{\pi \xi(2 T \pm 1)\}}{\sin (\pi \xi)} & (|\xi| \leqslant 1), \\ 0 & (|\xi|>1) .\end{cases}
$$

Proof. Relation (2.4) immediately follows from (2.2).

In order to prove (2.5), we first notice that

$$
\widehat{K}(\xi)=(1-|\xi|)^{+} \quad(\xi \in \mathbb{R}) .
$$

Moreover, as shown in Vaaler [29], the Fourier transform of $J(z):=\frac{1}{2} H^{\prime}(z)$ is given by

$$
\widehat{J}(\xi)=\left\{\begin{array}{lll}
\pi \xi(1-|\xi|) \cot (\pi \xi)+|\xi| & \text { if } & |\xi| \leqslant 1, \\
0 & \text { if } & |\xi|>1 .
\end{array}\right.
$$

We obtain (2.5) by replacing $b$ and $B$ by their definitions in (2.3) and appealing to (2.6) and (2.7).

The above result enables one to easily recover a classical result of Ingham [14. Since the precise form of the constants in (2.8) below plays an important rôle in the sequel, we provide a formal statement and a complete proof. 
Corollary 2.4. Let $\gamma>0$ be given and let $\left(\lambda_{n}\right)_{n \in \mathbb{Z}}$ denote a real sequence satisfying the condition

$$
\lambda_{n+1}-\lambda_{n} \geqslant \gamma>0 \quad(n \in \mathbb{Z}) .
$$

Then, for every interval $I$ of length $|I|=2 T / \gamma$, with $T>0$ and for every sequence $\left(a_{n}\right) \in \ell^{2}(\mathbb{Z}, \mathbb{C})$, we have

$$
\frac{2 T-1}{\gamma} \sum_{n \in \mathbb{Z}}\left|a_{n}\right|^{2} \leqslant \int_{I}\left|\sum_{n \in \mathbb{Z}} a_{n} \mathrm{e}\left(\lambda_{n} t\right)\right|^{2} \mathrm{~d} t \leqslant \frac{2 T+1}{\gamma} \sum_{n \in \mathbb{Z}}\left|a_{n}\right|^{2} .
$$

In particular, every interval $I$ of length $|I|>1 / \gamma$ is a domain associated to $\left(\lambda_{n}\right)$.

Proof. For $t \in \mathbb{R}$, let $\psi^{ \pm}(t):=\gamma m_{T}^{ \pm}(\gamma t)$, so that

$$
\widehat{\psi^{ \pm}}(\xi)=\widehat{m_{T}^{ \pm}}(\xi / \gamma) \quad(\xi \in \mathbb{R}) .
$$

By (2.5), this implies that $\widehat{\psi^{ \pm}}(\xi)=0$ whenever $|\xi|>\gamma$ and that $\widehat{\psi^{ \pm}}(0)=2 T \pm 1$. Therefore

$$
\int_{\mathbb{R}} \psi^{ \pm}(t)\left|\sum_{n \in \mathbb{Z}} a_{n} \mathrm{e}\left(\lambda_{n} t\right)\right|^{2} \mathrm{~d} t=\sum_{m, n \in \mathbb{Z}} a_{m} \overline{a_{n}} \widehat{\psi^{ \pm}}\left(\lambda_{m}-\lambda_{n}\right)=(2 T \pm 1) \sum_{n \in \mathbb{Z}}\left|a_{n}\right|^{2},
$$

from (2.5). Considering (2.4), we readily obtain the required bounds (2.8).

Remark 2.5. It is easy to see that the condition $|I|>1 / \gamma$ in the above statement is essentially sharp. Indeed, for $T<\frac{1}{2}$ and $\lambda_{n}=n$, one can choose $\left(a_{n}\right)$ as the sequence of Fourier coefficients of a function $f \in L^{2}\left[-\frac{1}{2}, \frac{1}{2}\right]$ which vanishes on $\left.\left[-\frac{1}{2}, \frac{1}{2}\right] \backslash\right]-T, T$. Actually, the condition is exactly sharp: Ingham provided in [14] a counterexample for the critical case $|I|=1 / \gamma$.

Remark 2.6. Vaaler gives in 29] a simple proof of Beurling's theorem according to which, if $F^{ \pm}(z)$ are functions of exponential type $2 \pi$ such that $F^{-}(x) \leqslant \operatorname{sgn}(x) \leqslant$ $F^{+}(x)$ for all real $x$, then

$$
\int_{\mathbb{R}}\left\{\operatorname{sgn}(x)-F^{-}(x)\right\} \mathrm{d} x \leqslant 1 \leqslant \int_{\mathbb{R}}\left\{F^{+}(x)-\operatorname{sgn}(x)\right\} \mathrm{d} x .
$$

Thus, the functions $B$ and $b$ are optimal. Selberg showed that this extremal property is shared by $m_{T}^{ \pm}$provided $2 T \in \mathbb{N}^{*}$. Thus, at least when $2 T / \gamma$ is an integer, the constants $(2 T \pm 1) / \gamma$ appearing in (2.8) are optimal in the framework of Ingham's method, as employed in the proof of Corollary 2.4 no better values may be derived by comparing $\mathbb{1}_{[-T / \gamma, T / \gamma]}$ to functions whose Fourier transforms vanish outside $[-\gamma, \gamma]$. When $2 T / \gamma$ is not an integer, the corresponding extremal problem has been solved by Logan [19. We shall not discuss this last case here.

\section{Some BACKGROUND ON NONHARMONIC FOURIER SERIES}

This section is devoted to recalling or establishing basic results on nonharmonic Fourier series which play an important rôle in the proofs of our main theorems. More precisely, we obtain several inequalities in the spirit of classical estimates of Beurling [4] and Kahane [16]; see also Baiocchi, Komornik, and Loreti 20. The main novelty brought in here resides in making the dependency of the involved constants explicit in terms of various parameters. 
Theorem 3.1. Let $\Lambda=\left(\lambda_{n}\right)_{n \in \mathbb{Z}}$ be a real sequence such that

$$
\gamma_{1}:=\inf _{n \in \mathbb{Z}}\left(\lambda_{n+1}-\lambda_{n}\right)>0,
$$

and, for some $p \in \mathbb{N}^{*}$,

$$
\gamma_{p}:=\inf _{n \in \mathbb{Z}}\left(\frac{\lambda_{n+p}-\lambda_{n}}{p}\right)>0 .
$$

Then, any open interval $I \subset \mathbb{R}$ of length $|I|>1 / \gamma_{p}$ is a domain associated to $\Lambda$. More precisely, for any $\gamma \in] 0, \gamma_{p}[$ and any interval I with length $|I| \geqslant 1 / \gamma$, there exists a constant $\kappa=\kappa\left(\gamma_{1}\right)>0$ such that, writing $\varepsilon:=\frac{1}{2}\left\{1 / \gamma-1 / \gamma_{p}\right\}$, we have

$$
\int_{I}|f(x)|^{2} \mathrm{~d} x \geqslant \frac{\kappa \varepsilon^{5 p+2}}{p^{12 p}} \sum_{n \in \mathbb{Z}}\left|a_{n}\right|^{2}
$$

for any sequence $\left(a_{n}\right) \in \ell^{2}(\mathbb{Z}, \mathbb{C})$ and $f$ as defined in (2.1).

The proof of Theorem 3.1 necessitates several lemmas. In order to state these, we consider $g \in] \gamma, \gamma_{p}[$. Then, under the assumptions of Theorem 3.1], each interval of length $p g$ contains at most $p$ values of the sequence $\Lambda$. Set

$$
J_{k}:=[k p g,(k+1) p g[\quad(k \in \mathbb{Z}) .
$$

By inserting, for all $n$, at most $m$ well-spaced points between $\lambda_{n}$ and $\lambda_{n+1}$, where $m \in \mathbb{N}^{*}$ is defined by $m \gamma_{1}<\lambda_{n+1}-\lambda_{n} \leqslant(m+1) \gamma_{1}$, we see that $\Lambda$ can be extended to a sequence, still denoted by $\Lambda$, satisfying the following conditions:

(A1): for all $k$, the interval $J_{k}$ contains exactly $p$ values of $\Lambda$;

(A2): $\inf _{n \in \mathbb{Z}}\left(\lambda_{n+1}-\lambda_{n}\right) \geqslant \frac{1}{2} \gamma_{1}>0$.

Therefore, without loss of generality, the assumptions of Theorem 3.1 may be replaced by conditions (A1) and (A2) above.

Occasionally, we will assume that the sequence $\Lambda$ satisfies the extra condition

(A3): $\min _{n}\left|\lambda_{n}\right| \geqslant \frac{1}{2} \gamma_{1}$.

In [4, Lemma 7, Section V] it has been shown that, under assumptions (A1)-(A3), the formula

$$
F(z):=\lim _{R \rightarrow \infty} \prod_{\left|\lambda_{n}\right|<R}\left(1-\frac{z}{\lambda_{n}}\right)
$$

defines an entire function of $z$, vanishing on $\Lambda$ and satisfying

$$
F(0)=1, \quad|F(z)| \leqslant C(1+|z|)^{5 p} \mathrm{e}^{\pi|y| / g},
$$

where $C>0$ depends only on $\gamma_{1}, g$ and $p$. Here and in the sequel, we implicitly define real numbers $x$ and $y$ by $z:=x+i y$.

The result below makes explicit the dependencies upon $p$ and $g$ of the constant $C$ appearing in (3.2).

Lemma 3.2. Assume that the sequence $\Lambda$ satisfies assumptions (A1)-(A3) above and that $F$ is defined by (3.1). Then, there exists a constant $c=c\left(\gamma_{1}\right)>0$ such that

$$
|F(z)| \leqslant \mathrm{e}^{c p}(1+|z|)^{5 p} \mathrm{e}^{\pi|y| / g} \quad(z \in \mathbb{C}) .
$$


Proof. We first establish an upper bound for $|F|$ on the positive real axis. We have

$$
|F(x)|=\prod_{k \in \mathbb{Z}} \prod_{\lambda_{n} \in J_{k}}\left|1-\frac{x}{\lambda_{n}}\right| \quad(x \geqslant 0) .
$$

Let $m \in \mathbb{N}$ be defined by $x \in J_{m}$. For $k \in \mathbb{Z}$ and $\lambda_{n} \in J_{k}$, we have

$$
\left|1-\frac{x}{\lambda_{n}}\right| \leqslant\left|1-\frac{x}{(k+1) p g}\right| \quad \text { if } k \leqslant-2 \text { or } k>m,
$$

and

$$
\left|1-\frac{x}{\lambda_{n}}\right| \leqslant\left|1-\frac{x}{k p g}\right| \quad \text { if } 1 \leqslant k<m .
$$

Using this fact and leaving the factors corresponding to $k=m, 0,-1$ in (3.4) unchanged, we obtain that, for every $x \geqslant 0$, we have

$$
\begin{aligned}
& |F(x)| \\
& \leqslant \prod_{k \neq 0}\left|1-\frac{x}{k p g}\right|^{p}\left\{\prod_{\lambda_{n} \in J_{-1} \cup J_{0} \cup J_{m}}\left|1-\frac{x}{\lambda_{n}}\right|\right\}\left|1-\frac{x}{m p g}\right|^{-p}\left|1-\frac{x}{(m+1) p g}\right|^{-p},
\end{aligned}
$$

if $m \neq 0$, and

$$
|F(x)| \leqslant \prod_{k \neq 0}\left|1-\frac{x}{k p g}\right|^{p}\left\{\prod_{\lambda_{n} \in J_{-1} \cup J_{0}}\left|1-\frac{x}{\lambda_{n}}\right|\right\}\left|1-\frac{x}{p g}\right|^{-p},
$$

if $m=0$.

For the sake of simplicity, let us assume henceforth that $m \neq 0$; the case $m=0$ can be tackled similarly. We then observe that, by Euler's product formula (see, for instance, Ahlfors [1, p.195]),

$$
\prod_{k \neq 0}\left|1-\frac{x}{k p g}\right|=\prod_{k \geqslant 1}\left|1-\frac{(x / p g)^{2}}{k^{2}}\right|=\left|\frac{\sin (\pi x / p g)}{\pi x / p g}\right| \quad(x \geqslant 0) .
$$

On the other hand, it follows from (A3) that

$$
\left|1-\frac{x}{\lambda_{n}}\right| \leqslant 1+\frac{x}{\lambda_{n}} \leqslant 1+\frac{2 x}{\gamma_{1}} \quad(x \geqslant 0) .
$$

The above estimate, combined with (3.5) and (3.6), yields that

$$
|F(x)| \leqslant\left|\frac{\sin (\pi x / p g)}{(\pi x / p g)}\right|^{p}\left(1+\frac{2 x}{\gamma_{1}}\right)^{3 p}\left|1-\frac{x}{m p g}\right|^{-p}\left|1-\frac{x}{(m+1) p g}\right|^{-p} .
$$

This last relation and the fact (easy to check) that, for a suitable absolute constant $C_{0}$, we have

$$
\sup _{t \in \mathbb{R}}\left|\frac{\sin (\pi t)}{\pi t\{1-t / m\}\{1-t /(m+1)\}}\right| \leqslant C_{0}(m+1)^{2} \leqslant C_{0}\left(1+\frac{x}{p g}\right)^{2},
$$

imply that

$$
|F(x)| \leqslant C_{0}^{p}\left(1+\frac{x}{p g}\right)^{2 p}\left(1+\frac{2 x}{\gamma_{1}}\right)^{3 p} \quad(x \geqslant 0) .
$$

Since $g \geqslant \gamma_{1}$, it follows that there exists $a_{0}=a_{0}\left(\gamma_{1}\right)>0$ such that

$$
|F(x)| \leqslant \mathrm{e}^{a_{0} p}(1+|x|)^{5 p} \quad(x \geqslant 0) .
$$

A symmetric treatment yields that (3.7) also holds for real negative $x$. 
Similarly, we easily deduce from the formula

$$
|F(i y)|^{2}=\prod_{n \in \mathbb{Z}}\left(1+y^{2} / \lambda_{n}^{2}\right) \quad(y \in \mathbb{R})
$$

and from Euler's product formula that, for all real $y$,

$$
|F(i y)| \leqslant\left(1+\frac{4 y^{2}}{\gamma_{1}^{2}}\right)^{p}\left|\frac{\sinh (\pi y / p g)}{(\pi y / p g)}\right|^{p} \leqslant \mathrm{e}^{b_{0} p}(1+|y|)^{5 p} \mathrm{e}^{\pi|y| / g},
$$

where $b_{0}=b_{0}\left(\gamma_{1}\right)$. By applying the Phragmén-Lindelöf principle (see, for instance, Titchmarsh [28, p.177]) to $F(z)(1+z)^{-5 p} \mathrm{e}^{i \pi z / g}$ in the first quadrant, we deduce that (3.3) holds on this domain with

$$
c:=\max \left(a_{0}, b_{0}\right) .
$$

A similar reasoning on the other three quadrants yields that (3.3) holds for every $z \in \mathbb{C}$.

Lemma 3.3. Let $\varepsilon>0$. Then, there exists $h \in C^{\infty}(\mathbb{R})$ such that $\operatorname{supp} h \subset[-\varepsilon, \varepsilon]$, $\widehat{h}(0)=1$ and

$$
|\widehat{h}(z)| \leqslant D \frac{p^{11 p} \mathrm{e}^{2 \pi \varepsilon|y|}}{\varepsilon^{5 p+2}(1+|z|)^{5 p+2}} \quad\left(z \in \mathbb{C}, p \in \mathbb{N}^{*}\right),
$$

where $D$ is an absolute constant.

Proof. Let $\sigma \in C^{\infty}(\mathbb{R})$ be defined by

$$
\sigma(x):= \begin{cases}\mathrm{e}^{-1 /\left(1-x^{2}\right)} & (|x| \leqslant 1) \\ 0 & (|x|>1)\end{cases}
$$

and consider the function

$$
h(x):=\frac{\sigma(x / \varepsilon)}{\varepsilon \int_{-1}^{1} \sigma(x) \mathrm{d} x} .
$$

It is clear that $h \in C^{\infty}(\mathbb{R}), \operatorname{supp} h \subset[-\varepsilon, \varepsilon]$ and $\widehat{h}(0)=1$. Moreover, according to [5], we have

$$
\left\|h^{(m)}\right\|_{\infty} \leqslant 3 \frac{\left(2^{m} m !\right)^{2}}{\varepsilon^{m+1}} \quad(m \in \mathbb{N}) .
$$

It follows that

$$
\sup _{x \in \mathbb{R}}\left|(2 \pi x)^{m} \widehat{h}(x)\right| \leqslant 3 \frac{\left(2^{m} m !\right)^{2}}{\varepsilon^{m+1}} \quad(m \in \mathbb{N}) .
$$

Furthermore, successive integrations by parts yield that, for all $z=x+i y \in \mathbb{C}$, we have

$$
\begin{aligned}
|\widehat{h}(z)|+\left|(2 \pi i z)^{5 p+2} \widehat{h}(z)\right| & \leqslant\left|\int_{-\varepsilon}^{\varepsilon} h(t) \mathrm{e}(t z) \mathrm{d} t\right|+\left|\int_{-\varepsilon}^{\varepsilon} h^{(5 p+2)}(t) \mathrm{e}(t z) \mathrm{d} t\right| \\
& \leqslant 6 \mathrm{e}^{2 \pi \varepsilon|y|}\left\{1+\frac{\left\{2^{5 p+2}(5 p+2) !\right\}^{2}}{\varepsilon^{5 p+2}}\right\} .
\end{aligned}
$$

The above inequality clearly implies the required conclusion via Stirling's formula. 
Proof of Theorem 3.1. For each $n \in \mathbb{Z}$, we define a function $F_{n}$ by applying the right-hand side of (3.1) to $z-\lambda_{n}$ for the sequence $\left(\lambda_{m}-\lambda_{n}\right)_{m \neq n}$ : indeed this sequence clearly satisfies assumptions (A1)-(A3). Therefore, by Lemma 3.2 we infer that

$$
F_{n}\left(\lambda_{n}\right)=1, \quad F_{n}\left(\lambda_{m}\right)=0 \quad(m \neq n), \quad\left|F_{n}(z)\right| \leqslant \mathrm{e}^{c p}\left(1+\left|z-\lambda_{n}\right|\right)^{5 p} \mathrm{e}^{\pi|y| / g},
$$

where $c$ is a constant depending only on $\gamma_{1}$. Let us write

$$
g_{n}(z):=F_{n}(z) \widehat{h}\left(z-\lambda_{n}\right) \quad(n \in \mathbb{Z}),
$$

where $h$ is the function defined in Lemma 3.3 with $\varepsilon:=\frac{1}{2}(1 / \gamma-1 / g)$.

From Lemma 3.3. we have

$$
g_{n}\left(\lambda_{n}\right)=1, \quad g_{n}\left(\lambda_{m}\right)=0 \quad(n \neq m), \quad\left|g_{n}(z)\right| \leqslant \frac{C_{1} p^{12 p} \mathrm{e}^{\pi|y| / \gamma}}{\varepsilon^{5 p+2}\left\{1+\left|z-\lambda_{n}\right|^{2}\right\}},
$$

where $C_{1}$ depends only on $\gamma_{1}$.

Thus, for each $n$, the entire function $g_{n}$ has exponential type $\pi / \gamma$ and its restriction to $\mathbb{R}$ is square-integrable. By the Paley-Wiener theorem (see, for instance, Rudin [24, p.375]) its Fourier transform has compact support included in $[-1 /(2 \gamma), 1 /(2 \gamma)]$.

The function

$$
\psi(z):=\sum_{n \in \mathbb{Z}} \overline{a_{n}} g_{n}(z)
$$

hence satisfies

$$
\int_{-1 /(2 \gamma)}^{1 /(2 \gamma)} \widehat{\psi}(\vartheta) f(\vartheta) \mathrm{d} \vartheta=\sum_{n \in \mathbb{Z}} a_{n} \int_{-1 /(2 \gamma)}^{1 /(2 \gamma)} \widehat{\psi}(\vartheta) \mathrm{e}\left(\lambda_{n} \vartheta\right) \mathrm{d} \vartheta=\sum_{n \in \mathbb{Z}} a_{n} \psi\left(\lambda_{n}\right)=\sum_{n \in \mathbb{Z}}\left|a_{n}\right|^{2} .
$$

Moreover,

$$
\begin{aligned}
\|\psi\|_{2}^{2} & =\sum_{m \in \mathbb{Z}} \sum_{n \in \mathbb{Z}} a_{m} \overline{a_{n}} \int_{\mathbb{R}} \overline{g_{m}(x)} g_{n}(x) \mathrm{d} x \\
& \leqslant \frac{C_{1}^{2} p^{24 p}}{\varepsilon^{10 p+4}} \sum_{m \in \mathbb{Z}} \sum_{n \in \mathbb{Z}}\left|a_{m} a_{n}\right| \int_{\mathbb{R}} \frac{\mathrm{d} x}{\left(1+\left|x-\lambda_{m}\right|^{2}\right)\left(1+\left|x-\lambda_{n}\right|^{2}\right)},
\end{aligned}
$$

where $C_{1}$ depends only on $\gamma_{1}$. The above estimate, combined with the elementary inequality

$$
\int_{\mathbb{R}} \frac{\mathrm{d} x}{\left(1+\left|x+\lambda_{m}\right|^{2}\right)\left(1+\left|x+\lambda_{n}\right|^{2}\right)} \leqslant \frac{4 \pi}{1+\left|\lambda_{m}-\lambda_{n}\right|^{2}} \quad\left(m, n \in \mathbb{N}^{*}\right),
$$

yields that

$$
\|\psi\|_{2}^{2} \leqslant \frac{C_{2} p^{24 p}}{\varepsilon^{10 p+4}} \sum_{m \in \mathbb{Z}} \sum_{n \in \mathbb{Z}} \frac{\left|a_{m} a_{n}\right|}{1+\left(\lambda_{m}-\lambda_{n}\right)^{2}},
$$

where $C_{2}$ depends only on $\gamma_{1}$. Since the sequence $\Lambda$ is regular, the last estimate implies that

$$
\|\psi\|_{2}^{2} \leqslant \frac{C_{3} p^{24 p}}{\varepsilon^{10 p+4}} \sum_{n \in \mathbb{Z}}\left|a_{n}\right|^{2},
$$

where $C_{3}$ only depends on $\gamma_{1}$. In view of (3.9), the above inequality furnishes the required conclusion. 
Definition 3.4. Let $\mathscr{S}$ be a countable set. A regular sequence $\Lambda=\left(\lambda_{s}\right)_{s \in \mathscr{S}} \subset \mathbb{R}^{2}$, with $\lambda_{s}=\left(\mu_{s}, \nu_{s}\right)$ is said to have regular projections if

$$
\varrho(\Lambda)=\min \left(\inf _{\substack{s, r \in \mathscr{S} \\ \mu_{s} \neq \mu_{r}}}\left|\mu_{s}-\mu_{r}\right|, \inf _{\substack{s, r \in \mathscr{S} \\ \nu_{s} \neq \nu_{r}}}\left|\nu_{s}-\nu_{r}\right|\right)>0 .
$$

We now state and prove a two-dimensional version of the Beurling-Kahane inequality.

Proposition 3.5. Let $\mathscr{S}$ be a countable set, let $\Lambda=\left(\lambda_{s}\right)_{s \in \mathscr{S}} \subset \mathbb{R}^{2}$, with $\lambda_{s}=$ $\left(\mu_{s}, \nu_{s}\right)$, be a sequence with regular projections and let $\mathcal{N} \subset \mathbb{R}$ denote the range of the sequence $\left(\nu_{s}\right)_{s \in \mathscr{S}}$. Assume that, for some $p, q \in \mathbb{N}^{*}$ and suitable $\gamma_{p}>0, \delta_{q}>0$, we have

$$
\begin{aligned}
& \sup _{s \in \mathscr{S}}\left|\left\{r \in \mathscr{S}: \nu_{r}=\nu_{s},\left|\mu_{r}-\mu_{s}\right| \leqslant \frac{1}{2} p \gamma_{p}\right\}\right| \leqslant p, \\
& \sup _{y \in \mathbb{R}}\left|\left\{\nu \in \mathcal{N}:|\nu-y| \leqslant \frac{1}{2} q \delta_{q}\right\}\right| \leqslant q .
\end{aligned}
$$

Then, for all intervals $I, J$ with lengths $|I|>1 / \gamma_{p},|J|>1 / \delta_{q}$, the set $I \times J$ is a domain associated to $\Lambda$. More precisely, if $|I|=(1+\varepsilon) / \gamma_{p},|J|=(1+\varepsilon) / \delta_{q}$, then there exists a constant $C=C(\varepsilon, \varrho(\Lambda))$ such that

$$
\int_{I} \mathrm{~d} x \int_{J}\left|\sum_{s \in \mathscr{S}} a_{s} \mathrm{e}\left(\mu_{s} x+\nu_{s} t\right)\right|^{2} \mathrm{~d} t \geqslant \frac{|I|^{7 p}|J|^{7 q}}{\mathrm{e}^{C(p+q)} p^{12 p} q^{12 q}} \sum_{s \in \mathscr{S}}\left|a_{s}\right|^{2} \quad\left(\left(a_{s}\right) \in \ell^{2}(\mathscr{S}, \mathbb{C})\right) .
$$

Proof. For $\left(a_{s}\right) \in \ell^{2}(\mathscr{S}, \mathbb{C})$, let $F: \mathbb{R}^{2} \rightarrow \mathbb{C}$ be defined by

$$
F(x, t)=\sum_{s \in \mathscr{S}} a_{s} \mathrm{e}\left(\mu_{s} x+\nu_{s} t\right) \quad\left((x, t) \in \mathbb{R}^{2}\right) .
$$

We may plainly write, alternatively,

$$
F(x, t)=\sum_{\nu \in \mathcal{N}} f_{\nu}(x) \mathrm{e}(\nu t)
$$

with

$$
f_{\nu}(x):=\sum_{\nu_{s}=\nu} a_{s} \mathrm{e}\left(\mu_{s} x\right) .
$$

By condition (3.11), Theorem 3.1 can be applied, for every $x$, to the partial function $t \mapsto F(x, t)$. Thus, there exists a constant $C_{1}$, depending only on $\varrho(\Lambda)$, such that

$$
\int_{J}|F(x, t)|^{2} \mathrm{~d} t \geqslant \frac{C_{1}}{\left(2 \delta_{q} / \varepsilon\right)^{7 q} q^{12 q}} \sum_{\nu \in \mathcal{N}}\left|f_{\nu}(x)\right|^{2} .
$$

Now, we appeal to condition (3.10) and apply Theorem 3.1 to each $f_{\nu}$, with $\nu \in \mathcal{N}$. It follows that there exists $C_{2}>0$, depending only on $\varrho(\Lambda)$, such that

$$
\int_{I}\left|f_{\nu}(x)\right|^{2} \mathrm{~d} x \geqslant \frac{C_{2}}{\left(2 \gamma_{p} / \varepsilon\right)^{7 p} p^{12 p}} \sum_{\nu_{s}=\nu}\left|a_{s}\right|^{2} .
$$

From (3.13) and (3.14), we deduce that $I \times J$ is a domain associated to $\Lambda$ and that (3.12) holds with, say, $C:=7 \ln (2 / \varepsilon)-\ln \max \left(C_{1}, C_{2}\right)$. 


\section{Proofs of the MAIN RESUlts}

An essential step in the proofs of Theorems 1.1 and 1.4 is the following result on the distribution of lattice points in the neighbourhood of an ellipse.

Theorem 4.1. Let $u, v>0$. For $M \in \mathbb{N}, N \in \mathbb{N}^{*}, R>0$, define the quantity $Z=Z(u, v ; M, N, R)$ by the formula

$$
Z:=\left|\left\{(m, n) \in \mathbb{Z}^{2}: M<m \leqslant M+N,\left|u m^{2}+v n^{2}-R^{2}\right|<1\right\}\right| .
$$

Then, there exists a real, positive sequence $\left(\varepsilon_{N}\right)_{N \in \mathbb{N}}$, possibly depending on $u$ and $v$, such that $\lim _{N \rightarrow \infty} \varepsilon_{N}=0$ and

$$
Z \leqslant \varepsilon_{N} N \quad\left(N \in \mathbb{N}^{*}\right) .
$$

Moreover, if $u / v \in \mathbb{Q}^{*}$, say $u=U / W, v=V / W$ with $U, V \in \mathbb{N}^{*}, W \geqslant 1$, we may choose $\varepsilon_{N}:=A W \ln _{2}(3 U V) \sqrt{\ln (2 N) / N}$, where the constant $A$ is absolute, whenever $N>N_{0}(U, V)$ with

$$
N_{0}(U, V):=\exp \left\{B\left(\ln _{2}(3 U V)\right)^{4}\right\}
$$

and $B$ is absolute. If $u / v \in \mathbb{R} \backslash \mathbb{Q}$, we can take $\varepsilon_{N}=C_{0} / \ln _{2} q_{N}$ where $C_{0}$ depends at most on $u$ and $v$ and $q_{N}$ is the largest denominator not exceeding $\sqrt{N}$ of a convergent of $u / v$.

We postpone the proof of this statement until Section 6

Temporarily accepting Theorem 4.1 we will show that Theorems 1.1 and 1.4 are comparatively simple consequences of the following result.

Theorem 4.2. Let $u, v \in \mathbb{R}_{+}^{*}$ and let $\mathscr{U} \subset \mathbb{R}^{2}$ be a nonempty open set. Then, there exists $\delta=\delta(\mathscr{U})=\delta(\mathscr{U} ; u, v)>0$ such that,

$$
\int_{\mathscr{U}}\left|\sum_{m, n \in \mathbb{Z}} a_{m n} \mathrm{e}\left(n x+\left(u m^{2}+v n^{2}\right) t\right)\right|^{2} \mathrm{~d} x \mathrm{~d} t \geqslant \delta(\mathscr{U}) \sum_{m, n \in \mathbb{Z}}\left|a_{m n}\right|^{2}
$$

for all sequences $\left(a_{m n}\right) \in \ell^{2}(\mathbb{Z} \times \mathbb{Z}, \mathbb{C})$.

Moreover, if $u=U / W, v=V / W$ with $U, V, \in \mathbb{N}^{*}, W \geqslant 1$, and $\mathscr{U}=I \times J$ with $|I|=1 / \alpha,|J|=1 / \beta, \alpha>1, \beta>1$, we can choose $\delta(\mathscr{U})$ in (4.3) such that for any $\varepsilon>0$ there exists $D=D(\varepsilon)$ with

$$
\delta(\mathscr{U}) \geqslant W \exp \left\{-\exp \left(A\left(\ln _{2} 3 U V\right)^{4}\right) \alpha W(\ln \alpha W)^{2}-\mathrm{e}^{C_{1} \beta^{2}}\right\},
$$

where $C_{1}:=D(U V)^{\varepsilon}$.

Proof. We may plainly assume that $\mathscr{U}=I \times J$, with $|I|=1 / \alpha,|J|=1 / \beta$, and $\alpha>2, \beta>2$ throughout. We distinguish two cases according to whether $u / v$ is or is not a rational number.

Case 1. $u / v \in \mathbb{Q}^{*}$. We can then assume, without loss of generality, that $u=U / W$, $v=V / W$ with $U, V \in \mathbb{N}^{*}, W \geqslant 1$. Moreover, we may also suppose that $u$ and $v$ are positive integers, i.e. $W=1$ : indeed, the general case reduces to this one by the change of variables $t=s W$.

Consider the sequence

$$
\Lambda:=\left(n, u m^{2}+v n^{2}\right)_{m, n \in \mathbb{Z}}
$$


It clearly has regular projections. The set $\mathcal{N}$ introduced in Proposition 3.5 is then given by

$$
\mathcal{N}:=\left\{\nu \in \mathbb{R}: \exists m, n \in \mathbb{N}, \nu=u m^{2}+v n^{2}\right\} .
$$

As will be shown in Theorem 5.3 below, for any given $\varepsilon>0$, we have

$$
\mid \mathcal{N} \cap] x, x+y] \mid \ll_{\varepsilon} \frac{y(u v)^{\varepsilon}}{\sqrt{\ln y}} \quad(x \in \mathbb{R}, y \geqslant 2) .
$$

Therefore assumption (3.11) of Proposition 3.5 holds, with $\delta_{q}:=2 \beta$, for some integer

$$
q \leqslant \exp \left\{C_{2}(u v)^{\varepsilon} \beta^{2}\right\}
$$

provided $C_{2}=C_{2}(\varepsilon)$ is large enough. Indeed, (4.7) implies that

$$
\sup _{y \in \mathbb{R}}|\{\nu \in \mathcal{N}:|\nu-y| \leqslant \beta q\}| \leqslant C_{3} \frac{2 \beta_{q}(u v)^{\varepsilon}}{\sqrt{\ln (2 \beta q)}},
$$

where $C_{3}=C_{3}(\varepsilon)$. This upper bound is clearly at most $q$ when $q$ equals the right-hand side of (4.8) and $C_{2}$ is suitably chosen in terms of $\varepsilon$.

In order to prove that the sequence $\Lambda$ also satisfies assumption (3.10) of Proposition 3.5, we recall the constants $A$ and $B$ appearing in Theorem 4.1 and we define

$$
N_{0}:=N_{0}(u, v)=\exp \left(C\left(\ln _{2} 3 u v\right)^{4}\right), \quad N_{1}:=N_{0} \alpha^{2} \ln \alpha,
$$

where $C$ is an absolute constant, with $C>B$, which will be specified later. Next, we put

$$
p:=\left\lfloor\frac{N_{1}}{2 \alpha}\right\rfloor+1, \quad \gamma_{p}:=2 \alpha,
$$

Since $p \gamma_{p} \geqslant N_{1}>N_{0}$, we may apply Theorem 4.1 to get that, for all $m_{0}, n_{0}$, we have

$$
\sum_{\substack{(m, n) \in \mathbb{Z}^{2} \\\left|n-n_{0}\right| \leqslant p \gamma_{p} \\ m^{2}+v n^{2}=u m_{0}^{2}+v n_{0}^{2}}} 1 \leqslant A \ln _{2}(3 u v) \sqrt{N_{1} \ln \left(2 N_{1}\right)} .
$$

We readily verify that, for a suitable choice of $C$, the above upper bound does not exceed $p$. Indeed, writing temporarily $h:=\ln _{2}(3 u v)$, it follows from (4.9) and (4.10) that $N_{0}=\exp \left(C h^{4}\right)$ and $p=\frac{1}{2} \exp \left(C h^{4}\right) \alpha \ln \alpha$. Consequently, we have

$$
A h \sqrt{N_{1} \ln \left(2 N_{1}\right)} \leqslant A h \mathrm{e}^{C h^{4} / 2} \alpha \sqrt{\ln \alpha}\left\{C h^{4}+2 \ln (2 \alpha)+\ln _{2} \alpha\right\}^{1 / 2} \leqslant \frac{1}{2} \mathrm{e}^{C h^{4}} \alpha \ln \alpha=p
$$
provided $C$ exceeds some absolute constant. From this and (4.11), it follows that assumption (3.10) of Proposition 3.5 is satisfied with $p$ and $\gamma_{p}$ chosen as in (4.10). Note that

$$
p \leqslant N_{0} \alpha \ln \alpha .
$$

We have thus shown that the sequence $\Lambda$ defined in (4.5) satisfies the assumptions in Proposition 3.5 with $\gamma_{p}=2 \alpha, \delta_{q}=2 \beta$ and with $p$ (respectively $q$ ) satisfying (4.12) (respectively (4.8)), so that $I \times J$ is a domain associated to the sequence $\Lambda$. Moreover, inserting the above bounds for $p$ and $q$ in (3.12) yields the estimate (4.4). 
Case 2. $u / v \in \mathbb{R} \backslash \mathbb{Q}$. We immediately observe that each element $\nu$ of the set $\mathcal{N}$ defined in (4.6) now has a unique representation in the form $\nu=u m^{2}+v n^{2}$ with $(m, n) \in \mathbb{N} \times \mathbb{N}$. We then define $m_{\nu}:=m, n_{\nu}:=n$. With this notation, an assertion equivalent to (4.3) is that $\mathscr{U}=I \times J$ is a domain associated to the regular sequence

$$
\Lambda:=\left\{\left( \pm n_{\nu}, \nu\right): \nu \in \mathcal{N}\right\}
$$

In other words, we aim to show that there exists $\delta(\mathscr{U})>0$ such that, for every almost-periodic function

$$
F(x, t):=\sum_{\nu \in \mathcal{N}}\left\{a_{\nu} \mathrm{e}\left(n_{\nu} x+\nu t\right)+b_{\nu} \mathrm{e}\left(-n_{\nu} x+\nu t\right)\right\},
$$

with $\left(a_{\nu}\right),\left(b_{\nu}\right) \in \ell^{2}(\mathcal{N}, \mathbb{C})$, we have

$$
\int_{\mathscr{U}}|F(x, t)|^{2} \mathrm{~d} x \mathrm{~d} t \geqslant \delta(\mathscr{U}) \sum_{\nu \in \mathcal{N}}\left\{\left|a_{\nu}\right|^{2}+\left|b_{\nu}\right|^{2}\right\} .
$$

For $r>0$, let us consider the intervals

$$
\left.\left.\left.\left.I_{k}:=\right]\left(2 k-\frac{1}{2}\right) r,\left(2 k+\frac{1}{2}\right) r\right], J_{k}:=\right]\left(2 k+\frac{1}{2}\right) r,\left(2 k+\frac{3}{2}\right) r\right] \quad(k \in \mathbb{N}) .
$$

We put

$$
A_{r}:=\bigcup_{k \in \mathbb{N}} I_{k}, \quad B_{r}:=\mathbb{R}^{+} \backslash A_{r}=\bigcup_{k \in \mathbb{N}} J_{k},
$$

and we divide $\Lambda$ into two subsequences

$$
\Lambda_{1}:=\left\{\left( \pm n_{\nu}, \nu\right): \nu \in \mathcal{N} \cap A_{r}\right\}, \quad \Lambda_{2}:=\left\{\left( \pm n_{\nu}, \nu\right): \nu \in \mathcal{N} \cap B_{r}\right\} .
$$

We shall show that $\Lambda_{1}$ and $\Lambda_{2}$ both have associated domains of the form $I \times J$, where $I$ is an arbitrary open interval and $J$ is an open interval of length $|J|>2 S / r$, where $S=S(I)$ is suitably chosen. To this end, let $S>\frac{1}{2}$ and put

$$
g(t):=m_{S}^{-}(r t), \quad G(t):=m_{S}^{+}(r t), \quad \Delta(t):=G(t)-g(t) \quad(t \in \mathbb{R}),
$$

where $m_{S}^{ \pm}$are the functions introduced in (2.3).

It follows from (2.5) that

$$
\widehat{g}(0)=\frac{2 S-1}{r}, \quad \widehat{G}(0)=\frac{2 S+1}{r}, \quad \widehat{\Delta}(0)=\frac{2}{r} .
$$

Put

$$
\begin{gathered}
f_{k}(x, t):=\sum_{\nu \in I_{k}}\left\{a_{\nu} \mathrm{e}\left(n_{\nu} x\right)+b_{\nu} \mathrm{e}\left(-n_{\nu} x\right)\right\} \mathrm{e}(\nu t) \quad(k \in \mathbb{N}), \\
f(x, t):=\sum_{k \in \mathbb{N}} f_{k}(x, t) .
\end{gathered}
$$

The above definition of $g$ and (2.4) imply that, setting $J:=[-S / r, S / r]$, we have

$$
g(t) \leqslant \mathbb{1}_{J}(t) \quad(t \in \mathbb{R})
$$

so that

$$
\begin{aligned}
& \int_{J}|f(x, t)|^{2} \mathrm{~d} t \geqslant \int_{\mathbb{R}}|f(x, t)|^{2} g(t) \mathrm{d} t \\
& =\sum_{j \in \mathbb{N}} \sum_{k \in \mathbb{N}} \sum_{\nu \in I_{j}} \sum_{\mu \in I_{k}}\left\{a_{\nu} \mathrm{e}\left(n_{\nu} x\right)+b_{\nu} \mathrm{e}\left(-n_{\nu} x\right)\right\}\left\{\bar{a}_{\mu} \mathrm{e}\left(-n_{\mu} x\right)+\bar{b}_{\mu} \mathrm{e}\left(n_{\mu} x\right)\right\} \widehat{g}(\mu-\nu) .
\end{aligned}
$$


From this, inequality (4.14) and the fact that, by (2.5), we have $\widehat{g}(\mu-\nu)=0$ whenever $\nu \in I_{j}, \mu \in I_{k}$ with $j \neq k$, it follows that

$$
\begin{aligned}
\int_{J}|f(x, t)|^{2} \mathrm{~d} t & \geqslant \sum_{k \in \mathbb{N}} \int_{\mathbb{R}}\left|f_{k}(x, t)\right|^{2} g(t) \mathrm{d} t \\
& =\sum_{k \in \mathbb{N}} \int_{\mathbb{R}}\left|f_{k}(x, t)\right|^{2} G(t) \mathrm{d} t-\sum_{k \in \mathbb{N}} \int_{\mathbb{R}}\left|f_{k}(x, t)\right|^{2} \Delta(t) \mathrm{d} t,
\end{aligned}
$$

Now observe that, by Theorem 4.1, for every $k \in \mathbb{N}$ and $\gamma>0$ there exists $q_{0}=$ $q_{0}(\gamma, r)$ such that

$$
\sup _{z \in \mathbb{R}}\left|\left\{\nu \in \mathcal{N} \cap I_{k}:\left|n_{\nu}-z\right| \leqslant \frac{1}{2} q \gamma\right\}\right| \leqslant q \quad\left(q \geqslant q_{0}\right) .
$$

By Theorem 3.1 this implies that there exists a constant $c=c(I)>0$ such that

$$
\int_{I}\left|f_{k}(x, t)\right|^{2} \mathrm{~d} x \geqslant c \sum_{\nu \in I_{k}}\left\{\left|a_{\nu}\right|^{2}+\left|b_{\nu}\right|^{2}\right\}
$$

Moreover, appealing to the upper bound of (2.8) with $\gamma:=1$ and $T:=\frac{1}{2}|I|$, we also have

$$
\int_{I}\left|f_{k}(x, t)\right|^{2} \mathrm{~d} x \leqslant(1+|I|) \sum_{\nu \in I_{k}}\left\{\left|a_{\nu}\right|^{2}+\left|b_{\nu}\right|^{2}\right\} .
$$

Thus, integrating (4.15) with respect to $x$ and using (4.13), we deduce that

$$
\int_{I \times J}|f(x, t)|^{2} \mathrm{~d} x \mathrm{~d} t \geqslant d \sum_{k \in \mathbb{N}} \sum_{\nu \in \mathcal{N} \cap I_{k}}\left\{\left|a_{\nu}\right|^{2}+\left|b_{\nu}\right|^{2}\right\}
$$

with

$$
d:=c \widehat{G}(0)-(1+|I|) \widehat{\Delta}(0)=\{c(2 S+1)-2-2|I|\} / r>0,
$$

provided $S>S(I):=(1+|I|) / c(I)$.

We have therefore established that every rectangle $I \times J$ with $|J|>2 S(I) / r$ is a domain associated to $\Lambda_{1}$, and a similar argument yields the same conclusion for $\Lambda_{2}$. Since $r$ may be chosen arbitrarily large, it follows that $I \times J$ is, for all nonempty open intervals $I$ and $J$, a domain associated to $\Lambda_{1}$ and $\Lambda_{2}$.

The required conclusion now follows from Proposition 2.2

Proof of Theorem 1.1. The eigenvalues of the Neumann Laplacian in $\Omega$ are $2 \pi \lambda_{m, n}$ with

$$
\lambda_{m, n}=\frac{\pi m^{2}}{2 a^{2}}+\frac{\pi n^{2}}{2 b^{2}} \quad(m, n \in \mathbb{N}) .
$$

A corresponding family of normalized eigenfunctions in $L^{2}(\Omega)$ is

$$
\varphi_{m, n}\left(x_{1}, x_{2}\right)=\frac{2}{\sqrt{a b}} \cos \left(\frac{\pi m x_{1}}{a}\right) \cos \left(\frac{\pi n x_{2}}{b}\right) \quad(m, n \in \mathbb{N}) .
$$

For $m, n \in \mathbb{N}^{*}$ we put $\psi_{m, n}:=\left\langle\psi, \varphi_{m, n}\right\rangle$, where $\langle\cdot, \cdot\rangle$ denotes the inner product in $L^{2}(\Omega)$. It is easy to check that the solution $w$ of (1.1) is given by

$$
w\left(x_{1}, x_{2}, t\right)=\sum_{m, n \in \mathbb{N}} \psi_{m, n} \mathrm{e}\left(\lambda_{m, n} t\right) \varphi_{m, n}\left(x_{1}, x_{2}\right) .
$$


With no loss of generality we may assume that $\Gamma \supset I_{1} \times\{0\}$ where $I_{1} \subset[0, a]$ is an interval with positive length. A simple calculation shows that for every $T>0$ we have

$$
\int_{0}^{T} \int_{\Gamma}|w|^{2} \mathrm{~d} \Gamma \mathrm{d} t \geqslant \frac{4}{a b} \int_{0}^{T} \int_{I_{1}}\left|\sum_{m, n \in \mathbb{N}} \psi_{m, n} \mathrm{e}\left(\lambda_{m, n} t\right) \cos \left(\frac{\pi m x_{1}}{a}\right)\right|^{2} \mathrm{~d} x_{1} \mathrm{~d} t .
$$

The claimed assertions follow from this and Theorem 4.2 .

Proof of Theorem 1.4. The eigenvalues of the Dirichlet Laplacian in $\Omega$ are $2 \pi \mu_{m, n}$ with

$$
\mu_{m, n}=\frac{\pi m^{2}}{2 a^{2}}+\frac{\pi n^{2}}{2 b^{2}} \quad\left(m, n \in \mathbb{N}^{*}\right),
$$

and a corresponding family of normalized eigenfunctions in $H_{0}^{1}(\Omega)$ is

$$
\Phi_{m, n}\left(x_{1}, x_{2}\right):=\frac{2 \sqrt{a b}}{\pi \sqrt{b^{2} m^{2}+a^{2} n^{2}}} \sin \left(\frac{m \pi x_{1}}{a}\right) \sin \left(\frac{n \pi x_{2}}{b}\right) \quad\left(m, n \in \mathbb{N}^{*}\right) .
$$

We first show that statement (S2) implies statement (S1). Indeed, if (S1) does not hold, then we may assume, without loss of generality, that $\Gamma \subset \Gamma_{1}$. Thus, for every $m \in \mathbb{N}^{*}$, we have

$$
\int_{\Gamma}\left|\frac{\partial \Phi_{m, 1}}{\partial \nu}\right|^{2} \mathrm{~d} \Gamma \leqslant \int_{\Gamma_{1}}\left|\frac{\partial \Phi_{m, 1}}{\partial \nu}\right|^{2} \mathrm{~d} \Gamma=\frac{4 a}{b\left(b^{2} m^{2}+a^{2}\right)} \int_{0}^{a} \sin ^{2}\left(\frac{m \pi x_{1}}{a}\right) \mathrm{d} x_{1} .
$$

Consequently,

$$
\lim _{m \rightarrow \infty} \int_{\Gamma}\left|\frac{\partial \Phi_{m, 1}}{\partial \nu}\right|^{2} \mathrm{~d} \Gamma=0
$$

It is easy to see that the above estimate contradicts (S2).

We next show that (S1) implies (S2). For $\psi \in H_{0}^{1}(\Omega)$ and $m, n \in \mathbb{N}^{*}$, we put $\psi_{m, n}:=\left\langle\psi, \Phi_{m, n}\right\rangle_{1}$, where $\langle\cdot, \cdot\rangle_{1}$ denotes the inner product in $H_{0}^{1}(\Omega)$, i.e.

$$
\langle f, g\rangle_{1}=\int_{\Omega} \nabla f \cdot \nabla g \mathrm{~d} x \quad\left(f, g \in H_{0}^{1}(\Omega)\right) .
$$

It is easy to check that the solution $w$ of (1.4) is given by

$$
w\left(x_{1}, x_{2}, t\right)=\sum_{m, n \in \mathbb{N}^{*}} \psi_{m, n} \mathrm{e}\left(\mu_{m, n} t\right) \Phi_{m, n}\left(x_{1}, x_{2}\right) .
$$

A simple calculation shows that

$$
\begin{gathered}
\int_{0}^{T} \int_{\Gamma}|y|^{2} \mathrm{~d} \Gamma \mathrm{d} t \geqslant \frac{4 a}{b} \int_{0}^{T} \int_{I_{1}}\left|\sum_{m, n \geqslant 1} \frac{n \psi_{m n}}{\sqrt{b^{2} m^{2}+a^{2} n^{2}}} \mathrm{e}\left(\mu_{m, n} t\right) \sin \left(\frac{m \pi x_{1}}{a}\right)\right|^{2} \mathrm{~d} x_{1} \mathrm{~d} t \\
+\frac{4 a}{b} \int_{0}^{T} \int_{I_{2}}\left|\sum_{m, n \geqslant 1} \frac{m \psi_{m n}}{\sqrt{b^{2} m^{2}+a^{2} n^{2}}} \mathrm{e}\left(\mu_{m, n} t\right) \sin \left(\frac{n \pi x_{2}}{b}\right)\right|^{2} \mathrm{~d} x_{2} \mathrm{~d} t .
\end{gathered}
$$

The required conclusions hence follow from Theorem 4.2 


\section{LOCAL DENSITY OF ELLIPTIC INTEGERS}

This section is devoted to proving (4.7). We need the following number theoretic lemma and a strong form of Selberg's sieve, stated in the sequel.

Recall that the Legendre symbol is the mapping from $\mathbb{Z}$ onto $\{-1,0,1\}$ defined by the formula

$$
\left(\frac{n}{p}\right):=\left\{\begin{array}{lll}
1 & \text { if } & n \in Q_{p}, \\
0 & \text { if } & p \mid n, \\
-1 & \text { if } \quad n \in(\mathbb{Z} / p \mathbb{Z})^{*} \backslash Q_{p},
\end{array} \quad(n \in \mathbb{Z}),\right.
$$

where $Q_{p}$ is the set of all integers which are congruent to a nonzero square modulo $p$. The Legendre symbol is a multiplicative homomorphism and satisfies Gauss' reciprocity law

$$
\left(\frac{q}{p}\right)=\left(\frac{p}{q}\right)(-1)^{(p-1)(q-1) / 4}
$$

for all pairs of distinct odd primes $p, q$. Moreover, we classically have, for odd primes $p$,

$$
\left(\frac{-1}{p}\right)=(-1)^{(p-1) / 2}, \quad\left(\frac{2}{p}\right)=(-1)^{\left(p^{2}-1\right) / 8},
$$

where the first formula follows from straightforward algebraic considerations and the latter is due to Euler.

Recall that a Dirichlet character $\chi: \mathbb{Z} \rightarrow \mathbb{C}$ to a given modulus $q \in \mathbb{N}^{*}$ is a completely multiplicative homomorphism such that $\chi(n)=0$ whenever $(n, q)>1$ and $|\chi(n)|=1$ when $(n, q)=1$.

Lemma 5.1. Let d be a positive squarefree integer. Then there exists a nonprincipal Dirichlet character $\chi_{D}$ with modulus $D$ dividing $4 d$ such that

$$
\left(\frac{-d}{p}\right)=\chi_{D}(p)
$$

for all primes $p$.

Proof. Write $d=2^{v} \prod_{q} q$, where $v=0$ or 1 and $q$ runs through the odd prime divisors of $d$. By the classical properties of the Legendre symbol recalled above, we have

$$
\begin{aligned}
\left(\frac{-d}{p}\right) & =\left(\frac{-1}{p}\right)\left(\frac{2}{p}\right)^{v} \prod_{q}\left(\frac{q}{p}\right)=(-1)^{(p-1) / 2+v\left(p^{2}-1\right) / 8} \prod_{q}\left(\frac{q}{p}\right) \\
& =(-1)^{(p-1) / 2+v\left(p^{2}-1\right) / 8} \prod_{q}\left(\frac{p}{q}\right)(-1)^{(p-1)(q-1) / 4} .
\end{aligned}
$$

It is easily checked that the mappings

$$
n \mapsto\left\{\begin{array}{ll}
(-1)^{v\left(n^{2}-1\right) / 8+(n-1) / 2+(n-1) \sum_{q}(q-1) / 2} & \text { if } 2 \nmid n, \\
0 & \text { if } 2 \mid n,
\end{array} \quad \text { and } \quad n \mapsto\left(\frac{n}{q}\right)\right.
$$

are Dirichlet characters, of respective moduli $2^{w}$ and $q$ with $0 \leqslant w \leqslant 2+v$. This is all we need.

We now state Selberg's sieve estimate in the form we shall use. Recall that the notation $p^{r} \| d$ means that $p^{r} \mid d$ but $p^{r+1} \nmid d$. 
Theorem 5.2 (Selberg [25). Let $M, N \in \mathbb{N}$ and let $\mathscr{A} \subset] M, M+N] \cap \mathbb{N}$. Assume that, for each prime power $p^{r}, \mathscr{A}$ is excluded from $w\left(p^{r}\right)$ residue classes modulo $p^{r}$ and, furthermore, that, for each $p$, the forbidden residue classes $\bmod p^{r}$ and $\bmod p^{s}$ are disjoint whenever $r \neq s$. Then, for each $Q>1$, we have

$$
|\mathscr{A}| \leqslant \frac{N+Q^{2}}{L}
$$

with

$$
L:=\sum_{d \leqslant Q} \prod_{p^{r} \| d}\left\{\frac{1}{\vartheta\left(p^{r}\right)}-\frac{1}{\vartheta\left(p^{r-1}\right)}\right\}, \quad \vartheta\left(p^{r}\right):=1-\sum_{1 \leqslant s \leqslant r} \frac{w\left(p^{s}\right)}{p^{s}} .
$$

Given two positive integers $u$ and $v$, we call an integer $\nu$ elliptic if it has at least a representation in the form $\nu=u m^{2}+v n^{2}$ for some integers $m, n$. We denote by $\mathcal{N}(u, v)$ the set of elliptic integers associated to a given pair $(u, v)$.

The following result is a specific application of Selberg's estimate (5.19) stated above. To our knowledge, it is new, even for $u=v=1$, inasmuch as it does not follow either from sieving by primes or from classical techniques used to obtain an asymptotic formula for the number of elliptic integers not exceeding a given bound.

Theorem 5.3. Let $\varepsilon>0$ be fixed. For all pairs $(u, v)$ of positive integers, we have

$$
\left.\left.Z(u, v ; x):=\sup _{y \in \mathbb{R}} \mid \mathcal{N}(u, v) \cap\right] y, y+x\right] \mid \ll \frac{x(u v)^{\varepsilon}}{\sqrt{\ln x}} \quad(x \geqslant 2) .
$$

Proof. Without loss of generality, we may assume that $u$ and $v$ are squarefree. In this case, consider $\nu \in \mathcal{N}:=\mathcal{N}(u, v)$ and a prime number $p$ not dividing $u v$. If $p \mid \nu$, then either $p \mid n$ and so $p^{2} \mid \nu$ or $\left(\frac{-u v}{p}\right)=1$. By Lemma 5.1, this last condition is equivalent to $\chi_{D}(p)=1$ for some nonprincipal Dirichlet character $\chi_{D}$ modulo a divisor $D$ of $4 u v$. Now, by the orthogonality property of characters, we have

$$
\sum_{1 \leqslant n \leqslant D} \chi_{D}(n)=0
$$

Since, by definition, $\chi_{D}(n)=0$ whenever $(n, D)>1$, this implies that $\chi_{D}(p)=1$ if, and only if, $p$ belongs to a set of congruence classes modulo $D$ containing exactly $\frac{1}{2} \varphi(D)$ elements, where $\varphi(D)$ denotes Euler's totient. Note that $\varphi(D)$ must be even since $D=2$ is impossible for there exists only one, principal, character to the modulus 2 .

Now assume that $p \mid u v$. If $p \mid(u, v)$, then $p \mid \nu$. Otherwise, let us suppose, for instance, that $p \mid v$ and $p \nmid u$. Then $\nu \equiv u m^{2}(\bmod p)$, and so $\nu$ belongs to $\frac{1}{2}(p+1)$ residue classes $\bmod p$. A symmetric conclusion holds if $p \mid u$ and $p \nmid v$.

Let $\mathscr{P}_{0}(u v)$ denote the set of all primes $p$ such that $p \mid u v$ and let $\mathscr{P}_{1}(u v)$ be the set of those $p$ satisfying $p \nmid u v$ and $\chi_{D}(p)=-1$. We have shown that, if $p \in \mathscr{P}_{0}(u v)$, then $\nu$ is restricted to at most $\frac{1}{2}(p+1)$ classes modulo $p$, and that, if $p \in \mathscr{P}_{1}(u v)$, then either $p \nmid \nu$ or $p^{2} \mid \nu$. Moreover $\mathscr{P}_{1}(u v)$ is a union of $\frac{1}{2} \varphi(D)$ congruence classes modulo $D$. We apply Theorem 5.2 to bound $Z(x):=Z(x ; u, v)$, selecting

$$
w\left(p^{r}\right):= \begin{cases}\frac{1}{2}(p-1) & \text { if } r=1 \text { and } p \in \mathscr{P}_{0}(u v), \\ p-1 & \text { if } r=2 \text { and } p \in \mathscr{P}_{1}(u v), \\ 0 & \text { in all other cases. }\end{cases}
$$


Put $\mathscr{P}(u v):=\mathscr{P}_{0}(u v) \cup \mathscr{P}_{1}(u v)$. With the above choice, we get

$$
\vartheta\left(p^{r}\right)= \begin{cases}(p-1) /(p+1) & \text { if } r=1 \text { and } p \in \mathscr{P}_{0}(u v), \\ 1-(p-1) / p^{2} & \text { if } r \geqslant 2 \text { and } p \in \mathscr{P}_{1}(u v), \\ 1 & \text { in all other cases. }\end{cases}
$$

Therefore

$$
\frac{1}{\vartheta\left(p^{r}\right)}-\frac{1}{\vartheta\left(p^{r-1}\right)}= \begin{cases}\frac{2}{p-1} & \text { if } r=1 \text { and } p \in \mathscr{P}_{0}(u v), \\ \frac{p-1}{p^{2}-p+1} \leqslant \frac{1}{p} & \text { if } r=2 \text { and } p \in \mathscr{P}_{1}(u v), \\ 0 & \text { in all other cases. }\end{cases}
$$

Denoting by $\mathbb{N}_{j}(u v)$ the set of positive integers all of whose prime factors belong to $\mathscr{P}_{j}(u v)(j=0,1)$ and selecting $Q:=\sqrt{x}$ in (5.19), we obtain

$$
Z(x) \leqslant \frac{2 x}{\mathscr{L}_{0}(x) \mathscr{L}_{1}(x)} \quad(x \geqslant 2),
$$

with

$$
\mathscr{L}_{0}(x):=\sum_{\substack{d \leqslant x^{1 / 8} \\ d \in \mathbb{N}_{0}(u v)}} \frac{2^{\omega(d)} \mu(d)^{2}}{\varphi(d)}, \quad \mathscr{L}_{1}(x):=\sum_{\substack{d \leq x^{1 / 8} \\ d \in \mathbb{N}_{1}(u v)}} \frac{\mu(d)^{2}}{d},
$$

where $\omega(d)$ denotes the number of distinct prime factors of $d, \varphi$ is Euler's function, and $\mu$ is the Möbius function.

For $x \geqslant(u v)^{8}$, we plainly have

$$
\begin{aligned}
\mathscr{L}_{0}(x) & \geqslant \sum_{d \mid u v} \frac{2^{\omega(d)} \mu(d)^{2}}{\varphi(d)}=\prod_{p \mid u v} \frac{p+1}{p-1} \\
& \geqslant \prod_{p \mid u v} \frac{1}{(1-1 / p)^{2}} \prod_{p}\left(1-\frac{1}{p^{2}}\right)=\frac{6(u v)^{2}}{\pi^{2} \varphi(u v)^{2}} .
\end{aligned}
$$

To estimate $\mathscr{L}_{1}(x)$ from below, we consider the sets

$$
\mathscr{C}^{ \pm}(D):=\left\{n \in \mathbb{N}: \chi_{D}(n)= \pm 1\right\},
$$

so $\mathscr{P}_{1}(u v)=\left\{p: p \nmid u v, p \in \mathscr{C}^{+}(D)\right\}$. Let $\mathscr{M}^{ \pm}(D)$ denote the set of positive integers all of whose prime factors belong to $\mathscr{C}^{ \pm}(D)$. Since any squarefree integer $n$ has a canonical representation in the form $n=r s t$ with $r \mid D, s \in \mathscr{M}^{+}(D)$, $t \in \mathscr{M}^{-}(D)$, we have

$$
\sum_{n \leqslant x^{1 / 8}} \frac{\mu(n)^{2}}{n} \leqslant \sum_{r \mid D} \frac{1}{r} \sum_{\substack{s \leqslant x^{1 / 8} \\ s \in \mathscr{M}^{+}(D)}} \frac{\mu(s)^{2}}{s} \sum_{\substack{t \leqslant x^{1 / 8} \\ t \in \mathscr{M}^{-}(D)}} \frac{\mu(t)^{2}}{t} .
$$

The $n$-sum is classically $\asymp \ln x$. Every summand in the $t$-sum may be further decomposed as $t=m d$ where $m \mid u v$ and $d \in \mathbb{N}_{1}(u v)$. Moreover each product $r m$ is 
a divisor of $u v$. Therefore

$$
\begin{aligned}
\ln x & \ll \sum_{w \mid u v} \frac{\mu(w)^{2}}{w} \mathscr{L}_{1}(x) \sum_{\substack{s \leqslant x^{1 / 8} \\
s \in \mathscr{M}^{+}(D)}} \frac{\mu(s)^{2}}{s} \leqslant \prod_{p \mid u v}\left(1+\frac{1}{p}\right) \mathscr{L}_{1}(x) \prod_{\substack{p \leqslant x \\
p \in \mathscr{C}^{+}(D)}}\left(1+\frac{1}{p}\right) \\
& \ll \frac{u v}{\varphi(u v)} \mathscr{L}_{1}(x) \exp \left\{\sum_{\substack{p \leqslant x \\
p \in \mathscr{C}^{+}(D)}} \frac{1}{p}\right\},
\end{aligned}
$$

where $\varphi$ denotes Euler's totient function. Now

$$
\sum_{\substack{p \leqslant x \\ p \in \mathscr{C}^{+}(D)}} \frac{1}{p}=\sum_{p \leqslant x} \frac{1+\chi_{D}(p)}{2 p}=\frac{1}{2} \ln _{2} x+\frac{1}{2} \sum_{p \leqslant x} \frac{\chi_{D}(p)}{p}+O(1),
$$

where the remainder is bounded by an absolute constant.

The last sum over $p$ may be estimated by the Siegel-Walfisz theorem; see, for instance, [8, ch. 22]. We obtain that, for any given $\varepsilon>0$, we have

$$
\sum_{p \leqslant x} \chi_{D}(p) \ll x \mathrm{e}^{-c \sqrt{\ln x}} \quad\left(x>\exp D^{\varepsilon}\right)
$$

for a suitable positive constant $c=c(\varepsilon)$. Estimating the sum trivially when $x \leqslant$ $\exp D^{\varepsilon}$ and using partial summation otherwise, we obtain

$$
\left|\sum_{p \leqslant x} \frac{\chi_{D}(p)}{p}\right| \leqslant \varepsilon \ln D+O(1) .
$$

Gathering our estimates, we arrive at

$$
\mathscr{L}_{1}(x) \gg_{\varepsilon} \frac{\varphi(u v)}{u v D^{\varepsilon}} \sqrt{\ln x} .
$$

Inserting this last estimate and (5.23) in (5.21) immediately yields the required bound (5.20) when $x>(u v)^{8}$. However, the result holds trivially when $x \leqslant(u v)^{8}$.

\section{Proof of Theorem 4.1}

Here, we establish inequality (4.1). We made no effort to optimize the bound for $\varepsilon_{N}$ as a function of $q_{N}$ when $u / v$ is irrational. It is likely that a refined technique will enable a significant improvement of this aspect of the result.

We start with a simple lemma in Diophantine Approximation. As is usual in this theory, we introduce the notation

$$
\|\vartheta\|:=\min _{n \in \mathbb{Z}}|\vartheta-n| \quad(\vartheta \in \mathbb{R}) .
$$

For all $\vartheta \in \mathbb{R}, Q \in \mathbb{N}^{*}$ we have, by Dirichlet's theorem,

$$
\mu(\vartheta ; Q):=\min _{1 \leqslant m \leqslant Q}\|m \vartheta\| \leqslant 1 / Q .
$$

Put

$$
q(\vartheta ; Q):=\min \{q: 1 \leqslant q \leqslant Q,\|q \vartheta\|=\mu(\vartheta ; Q)\} .
$$


For notational convenience, we extend the definition of $t \mapsto q(\vartheta ; t)$ to $[1, \infty[$ by setting $q(\vartheta ; t):=q(\vartheta ;[t])$. The integers $q(\vartheta ; t)$ describe the set of denominators of the convergents of $\vartheta$, viz.

$$
\left\{q:\|q \vartheta\|<\min _{1 \leqslant r<q}\|r \vartheta\|\right\} .
$$

The following result is analogous to Lemma 6.2 of 9 and can be proved by the same method. For the convenience of the reader, we recall the details.

Lemma 6.1. Let $\vartheta \in \mathbb{R}, Q \in \mathbb{N}^{*}, \nu \in \mathbb{N}^{*}, d \in \mathbb{N}^{*}, q:=q(\vartheta ; Q), q^{*}:=$ $q(\nu \vartheta / d ; 2 d Q)$. Then we have $q^{*} \geqslant q /(2 \nu)$.

Proof. There exist integers $r$ and $s$ such that $(r, q)=\left(s, q^{*}\right)=1$ and

$$
\left|\vartheta-\frac{r}{q}\right| \leqslant \frac{1}{q Q}, \quad\left|\frac{\nu \vartheta}{d}-\frac{s}{q^{*}}\right| \leqslant \frac{1}{2 d q^{*} Q} .
$$

It follows that

$$
\left|\frac{r}{q}-\frac{d s}{\nu q^{*}}\right| \leqslant \frac{1}{Q}\left\{\frac{1}{q}+\frac{1}{2 \nu Q^{*}}\right\} .
$$

If $r / q=d s /\left(\nu q^{*}\right)$, then $s / q^{*}=(\nu r) /(d q)$ and, reducing the fraction, we get

$$
q^{*}=q d /(\nu r, d q) \geqslant q d /\{(\nu, d q)(r, d)\} \geqslant q /(\nu, d q) \geqslant q / \nu .
$$

Otherwise, we have

$$
\frac{1}{\nu q q^{*}} \leqslant \frac{1}{Q}\left\{\frac{1}{q}+\frac{1}{2 \nu q^{*}}\right\}
$$

whence

$$
Q \leqslant \nu q^{*}+\frac{1}{2} q \leqslant \nu q^{*}+\frac{1}{2} Q .
$$

This yields the stated inequality.

We now embark on proving our theorem. Put $\vartheta:=u / v$. We employ distinct arguments according as $\vartheta$ is or is not a rational number.

Let us first consider the situation when $\vartheta \in \mathbb{Q}^{*}$. We then assume that $u \in \mathbb{N}^{*}$, $v \in \mathbb{N}^{*}$ since the general case easily follows from this.

Put $I:=] M, M+N]$. We have

$$
Z \leqslant 2 \sup _{k \in \mathbb{N}} Z_{k}
$$

with

$$
Z_{k}:=\left|\left\{(m, n) \in \mathbb{Z}^{2}: m \in I, u m^{2}+v n^{2}=k\right\}\right| .
$$

We shall show that the bound

$$
Z_{k} \ll \ln _{2}(3 u v) \sqrt{N \log (2 N)} \quad\left(N>N_{0}(u v)\right),
$$

where the implicit constant is absolute, holds uniformly with respect to $k \in \mathbb{N}$. This generalizes Theorem 7.4 in [22].

Recall the definition (5.18) of the Legendre symbol. It follows for instance from theorem 7.8.2 of 13 that, for each prime $p$ not dividing kuv, we have

$$
\sum_{0 \leqslant m<p}\left(\frac{k v-u v m^{2}}{p}\right)=-\left(\frac{u v}{p}\right) .
$$

Let $\mathscr{P}$ denote the set of prime numbers, set $\mathscr{P}_{3,4}:=\{p \in \mathscr{P}: p \equiv 3(\bmod 4)\}$ and

$$
\mathscr{P}_{3,4}(d):=\mathscr{P}_{3,4} \cap\{p \in \mathscr{P}: p \nmid d\} \quad(d \geqslant 1) .
$$


If the pair $(m, n)$ is counted by $Z_{k}$ and if $p \mid k$, then $u v m^{2} \equiv-(v n)^{2}(\bmod p)$. Since -1 is not a square modulo $p$, this implies $p \mid u v m^{2}$ and hence $p \mid m$. Thus, when $p \in \mathscr{P}_{3,4}(u v), p \mid k$, the abscissas $m$ of the elements of $Z_{k}$ are confined to a unique residue class modulo $p$.

If, on the contrary, $p \nmid k$, we have

$$
\left(\frac{k v-u v m^{2}}{p}\right) \in\{0,1\}
$$

whenever the pair $(m, n)$ is counted by $Z_{k}$. This implies that $m$ is restricted to at most

$$
1+\frac{1}{2} \sum_{0 \leqslant m<p}\left\{1+\left(\frac{k v-u v m^{2}}{p}\right)\right\} \leqslant \frac{1}{2}(p+3)
$$

residue classes modulo $p$, where the inequality follows from (6.4).

We have therefore established that, for each prime $p \in \mathscr{P}_{3,4}(u v)$, the abscissas $m$ of the pairs counted by $Z_{k}$ are excluded from at least $\frac{1}{2}(p-3)$ residue classes $p$. Applying Selberg's sieve as stated in Theorem 5.2 with

$$
w\left(p^{r}\right):= \begin{cases}\frac{1}{2}(p-3) & \text { if } r=1, p>5 \text { and } p \in \mathscr{P}_{3,4}(u v), \\ 0 & \text { in all other cases }\end{cases}
$$

we obtain

$$
Z_{k} \leqslant \frac{2 N}{\mathscr{L}_{N}}
$$

with

$$
\mathscr{L}_{N}:=\sum_{\substack{d \leqslant \sqrt{N} \\ d \in \mathscr{B}(u v)}} f(d),
$$

where $f(d):=\prod_{p \mid d}(p-6) /(p+6)$ and $\mathscr{B}(u v)$ denotes the set of squarefree integers $d$, all of whose prime factors lie in $\mathscr{P}_{3,4}(u v) \cap\{p: p \geqslant 7\}=\mathscr{P}_{3,4}(21 u v)$.

We need a lower bound for $\mathscr{L}_{N}$. To achieve this, we observe that we have the following Eulerian decomposition of Dirichlet series:

$$
\begin{aligned}
F(s) & :=\sum_{d \in \mathscr{B}(u v)} \frac{f(d)}{d^{s}}=\prod_{p \in \mathscr{P}_{3,4}(21 u v)}\left(1+\frac{f(p)}{p^{s}}\right) \\
& =\prod_{p \in \mathscr{P}_{3,4}}\left(1-\frac{1}{p^{s}}\right)^{-1} G_{u v}(s) H_{u v}(s),
\end{aligned}
$$

where

$G_{u v}(s):=\prod_{\substack{p \in \mathscr{P} \\ p \mid 21 u v}}\left(1-\frac{1}{p^{s}}\right)^{-1}, \quad H_{u v}(s):=\prod_{p \in \mathscr{P}_{3,4}(21 u v)}\left(1-\frac{12}{p^{s}(p+6)}-\frac{p-6}{(p+6) p^{2 s}}\right)$.

Introducing the unique nonprincipal character $\chi_{3,4}$ modulo 4 , defined by

$$
\chi_{3,4}(2 m+1)=(-1)^{m} \quad(m \geqslant 0),
$$

and the corresponding Dirichlet $L$-function, we further note that

$$
\prod_{p \in \mathscr{P}_{3,4}}\left(1-\frac{1}{p^{s}}\right)^{-1}=\left(1-2^{-s}\right)^{1 / 2} \zeta(s)^{1 / 2} L\left(s, \chi_{3,4}\right)^{-1 / 2} \prod_{p \in \mathscr{P}_{3,4}}\left(1-p^{-2 s}\right)^{-1 / 2} .
$$


We are thus in a position to estimate $\mathscr{L}_{N}$ by a Selberg-Delange type formula. From Theorem II.5.5 of [27], we readily obtain, with a suitable constant $K$,

$$
\mathscr{L}_{N}=K \frac{\varphi(u v)}{u v} \sqrt{\frac{N}{\ln N}}\left\{1+O\left(\frac{\left\{\ln _{2}(3 u v)\right\}^{4}}{\ln N}\right)\right\} .
$$

Here, we have used the bound

$$
\sum_{d \mid n} \frac{\mu(d)^{2}(\ln d)^{m}}{d} \ll_{m}\left(\ln _{2} 3 n\right)^{m+1} \quad(n \geqslant 1)
$$

proved in [10, equation (10)].

It follows that, for suitably large $C_{1}$ and $N_{0}(u v)$ as defined in (4.2), we have

$$
\mathscr{L}_{N} \gg \frac{\varphi(u v)}{u v} \sqrt{\frac{N}{\ln N}} \quad\left(N>N_{0}(u v)\right) .
$$

In view of (6.5), this yields (6.3), as required.

We now turn our attention to the case $\vartheta \in \mathbb{R} \backslash \mathbb{Q}$. We denote by $\langle x\rangle:=x-\lfloor x\rfloor$ the fractional part of a real number $x$ and let $g: \mathbb{R} \rightarrow \mathbb{Z}$ be the function defined by

$$
g(x)= \begin{cases}\lfloor x\rfloor & \text { if } 0 \leqslant\langle x\rangle<\frac{1}{2}, \\ \lfloor x\rfloor+1 & \text { if } \frac{1}{2} \leqslant\langle x\rangle<1 .\end{cases}
$$

Denote by $\mathscr{Q}$ the set of perfect squares. We may restrict ourselves to estimating, uniformly in $V>0$, the quantity

$$
E_{N}:=\sum_{\substack{m \in I^{2} \\ g\left(V-\vartheta m^{2}\right) \in \mathscr{Q}}} 1 .
$$

Let $y \geqslant 2$. The arithmetic multiplicative function $\lambda_{y}$ defined on prime powers $p^{r}$ by

satisfies

$$
\lambda_{y}\left(p^{r}\right):= \begin{cases}(-1)^{r} & \text { if } r=1 \text { or } 2 \text { and } p \leqslant y \\ 0 & \text { if } r \geqslant 3 \text { or } p>y\end{cases}
$$

$$
\mathbb{1}_{\mathscr{Q}}(n) \leqslant \sum_{d \mid n} \lambda_{y}(d) \quad(n \geqslant 1) .
$$

Moreover, we have $\lambda_{y}(n)=0$ whenever $n>N_{y}:=\prod_{p \leqslant y} p^{2}$. Letting $T\left(N_{y}\right)$ designate the total number of divisors of $N_{y}$, we have trivially

$$
T\left(N_{y}\right) \leqslant 3^{y} .
$$

Let $P(d)$ denote the largest prime factor of a natural integer $d$, with the convention that $P(1)=1$. It follows from the above considerations that

$$
E_{N} \leqslant \sum_{m \in I} \sum_{d \mid g\left(V-\vartheta m^{2}\right)} \lambda_{y}(d)=\sum_{P(d) \leqslant y} \lambda_{y}(d) \sum_{\substack{m \in I \\ g\left(V-\vartheta m^{2}\right) \equiv 0(\bmod d)}} 1 .
$$

Now $g\left(V-\vartheta m^{2}\right) \equiv 0(\bmod d)$ if, and only if, there exist $k \in \mathbb{Z}$ and $\eta \in\left[-\frac{1}{2}, \frac{1}{2}[\right.$ such that $V-\vartheta m^{2}=k d+\eta$, i.e.

$$
-\frac{1}{2 d} \leqslant \frac{V-\vartheta m^{2}}{d}<\frac{1}{2 d} \quad(\bmod 1) .
$$


If $D_{N}(d)$ denotes the discrepancy of the sequence $\left\{\vartheta m^{2} / d\right\}_{m \in I}$, we thus have

$$
\sum_{\substack{m \in I \\ g\left(V-\vartheta m^{2}\right) \equiv 0(\bmod d)}} 1=\frac{N}{d}+O\left(N D_{N}(d)\right) .
$$

From the Erdős-Turán inequality [11, [12] (see 23] for recent considerations upon optimal constants), we have for all $H \geqslant 1$,

$$
\left|D_{N}(d)\right| \leqslant \frac{1}{H}+\sum_{1 \leqslant \nu \leqslant H} \frac{\left|S_{\nu}(d)\right|}{\nu},
$$

where we have put

$$
S_{\nu}(d):=\frac{1}{N} \sum_{m \in I} \mathrm{e}\left(\vartheta \nu m^{2} / d\right)
$$

To estimate $S_{\nu}(d)$, we apply a classical inequality of Weyl (see for example [21], chap. 2, th. 1) stating that, for $\alpha \in \mathbb{R}, a \in \mathbb{Z}, q \in \mathbb{N}^{*},|\alpha-a / q| \leqslant 1 / q^{2}$, we have

$$
\sum_{m \in I} \mathrm{e}\left(\alpha m^{2}\right) \ll \frac{N}{\sqrt{q}}+\sqrt{(N+q) \ln q} .
$$

We bound $S_{\nu}(d)$ in terms of good rational approximations of the real number $\vartheta$. Let $q_{N}:=q(\vartheta ; \sqrt{N})$. From Lemma 6.1, we know that $q(\nu \vartheta / d ; N) \geqslant q_{N} /(2 \nu)$ for $1 \leqslant d \leqslant \frac{1}{2} \sqrt{N}, \nu \geqslant 1$.

By (6.9), it follows that

$$
S_{\nu}(d) \ll \sqrt{\nu / q_{N}}
$$

uniformly for $1 \leqslant \nu \leqslant q_{N}, 1 \leqslant d \leqslant \frac{1}{2} \sqrt{N}$.

Applying (6.8) with $H:=q_{N}^{1 / 3}$, we obtain

$$
D_{N}(d) \ll q_{N}^{-1 / 3} \quad\left(1 \leqslant d \leqslant \frac{1}{2} \sqrt{N}\right) .
$$

We now insert this inequality back into (6.7) and then (6.6). Taking the formula

$$
\sum_{P(d) \leqslant y} \frac{\lambda_{y}(d)}{d}=\prod_{p \leqslant y}\left(1-\frac{1}{p}+\frac{1}{p^{2}}\right) \ll \frac{1}{\ln y} \quad(y \geqslant 2)
$$

into account, we get

$$
E_{N} \ll \frac{N}{\ln y}+\frac{N 3^{y}}{q_{N}^{1 / 3}} .
$$

Selecting, for instance, $y=\frac{1}{4} \log 3 q_{N}$, we obtain the bound

$$
E_{N} \ll \frac{N}{\ln _{2} 3 q_{N}} .
$$

\section{REFERENCES}

1. L. V. Ahlfors, Complex analysis, third ed., McGraw-Hill Book Co., New York, 1978, An introduction to the theory of analytic functions of one complex variable, International Series in Pure and Applied Mathematics. MR510197 (80c:30001)

2. C. Baiocchi, V. Komornik, and P. Loreti, Ingham-Beurling type theorems with weakened gap conditions, Acta. Math. Hungar. 97 (2002), no. 1-2, 55-95. MR1932795 (2003i:42011)

3. C. Bardos, G. Lebeau, and J. Rauch, Sharp sufficient conditions for the observation, control and stabilization of waves from the boundary, SIAM J. Control. and Optim. 30 (1992), 10241065. MR1178650 (94b:93067) 
4. A. Beurling, Mittag-leffler lectures on harmonic analysis, in The collected works of Arne Beurling, Vol. 2, Contemporary Mathematicians, Birkhäuser Boston Inc., Boston, MA, 1989, Harmonic analysis, Edited by L. Carleson, P. Malliavin, J. Neuberger and J. Wermer. MR 1057614 (92k:01046b)

5. E. Bombieri, J. B. Friedlander, and H. Iwaniec, Primes in arithmetic progressions to large moduli. II, Math. Ann. 277 (1987), no. 3, 361-393. MR891581 (88f:11085)

6. N. Burq and M. Zworski, Geometric control in the presence of a black box, J. Amer. Math. Soc. 17 (2004), no. 2, 443-471. MR2051618

7. G. Chen, S. A. Fulling, F. J. Narcowich, and S. Sun, Exponential decay of energy of evolution equations with locally distributed damping, SIAM J. Appl. Math. 51 (1991), no. 1, 266-301. MR 1089141 (91k:35037)

8. H. Davenport, Multiplicative number theory, third ed., Graduate Texts in Mathematics, vol. 74, Springer-Verlag, New York, 2000, Revised and with a preface by Hugh L. Montgomery. MR:1790423 (2001f:11001)

9. R. de la Bretèche and G. Tenenbaum, Séries trigonométriques à coefficients arithmétiques, J. Anal. Math. 92 (2004), 1-79. MR.2072741(2005i:11103)

10. Y. Dupain, R. R. Hall, and G. Tenenbaum, Sur l'équirépartition modulo 1 de certaines fonctions de diviseurs, J. London Math. Soc. (2) 26 (1982), no. 3, 397-411. MR684553 (84m:10047)

11. P. Erdös and P. Turán, On a problem in the theory of uniform distribution. I, Nederl. Akad. Wetensch., Proc. 51 (1948), 1146-1154 = Indagationes Math. 10, 370-378 (1948). MR0027895 $(10: 372 c)$

12. , On a problem in the theory of uniform distribution. II, Nederl. Akad. Wetensch., Proc. 51 (1948), 1262-1269 = Indagationes Math. 10, 406-413 (1948). MR0027896 (10:372d)

13. L. K. Hua, Introduction to number theory, Springer, Berlin, Heidelberg, New York, 1982. MR:83f:10001

14. A. E. Ingham, Some trigonometrical inequalities with applications to the theory of series, Math. Zeitschrift 41 (1936), 367-379. MR1545625

15. S. Jaffard, Contrôle interne exact des vibrations d'une plaque rectangulaire., Port. Math. 47 (1990), no. 4, 423-429 (French). MR1090480(91j:93051)

16. J.-P. Kahane, Pseudo-périodicité et séries de Fourier lacunaires, Ann. Sci. École Norm. Sup. (3) 79 (1962), 93-150. MR27:4019

17. V. Komornik, On the exact internal controllability of a Petrowsky system, J. Math. Pures Appl. (9) 71 (1992), no. 4, 331-342. MR1176015 (93j:93019)

18. G. Lebeau, Contrôle de l'équation de Schrödinger, J. Math. Pures Appl. (9) 71 (1992), no. 3, 267-291. MR93i:35018

19. B. F. Logan, Bandlimited functions bounded below over an interval, Notices Amer. Math. Soc. 24 (1977), A-331.

20. L. Miller, How violent are fast controls for Schrödinger and plate vibrations?, Arch. Ration. Mech. Anal. 172 (2004), no. 3, 429-456. MR2062431|(2005d:93022)

21. H. L. Montgomery, Ten lectures on the interface between analytic number theory and harmonic analysis, CBMS Regional Conference Series in Mathematics, vol. 84, Published for the Conference Board of the Mathematical Sciences, Washington, DC, 1994. MR 1297543 (96i:11002)

22. K. Ramdani, T. Takahashi, G. Tenenbaum, and M. Tucsnak, A spectral approach for the exact observability of infinite-dimensional systems with skew-adjoint generator, J. Funct. Anal. 226 (2005), no. 1, 193-229. MR2158180 (2006g:93017)

23. J. Rivat and G. Tenenbaum, Constantes d'Erdös-Turán, Ramanujan J. 9 (2005), no. 1-2, 111-121. MR2166382 (2006g:11158)

24. W. Rudin, Real and complex analysis, third ed., McGraw-Hill Book Co., New York, 1987. MR 924157 (88k:00002)

25. A. Selberg, Remarks on multiplicative functions, Number theory day (Proc. Conf., Rockefeller Univ., New York, 1976), Springer, Berlin, 1977, pp. 232-241. Lecture Notes in Math., Vol. 626. MR0485750(58:5562)

26. G. Tenenbaum and M. Tucsnak, New blow-up rates for fast controls of Schrödinger and heat equations, Journal of Differential Equations 243 (2007), 70-100. MR2363470 
27. G. Tenenbaum, Introduction to analytic and probabilistic number theory, Cambridge Studies in Advanced Mathematics., vol. 46, Cambridge Univ. Press, Cambridge, 1995. MR 1342300 (97e:11005b)

28. E. C. Titchmarsh, The theory of functions, second ed., Oxford University Press, 1939 (English).

29. J. D. Vaaler, Some extremal functions in Fourier analysis, Bull. Amer. Math. Soc. (N.S.) 12 (1985), no. 2, 183-216. MR776471 (86g:42005)

Institut Élie Cartan, Université Henri Poincaré Nancy 1, BP 239, 54506 VandeuvreLÈs-NANCY, FRANCE

Institut Élie Cartan, Université Henri Poincaré Nancy 1, BP 239, 54506 VandeuvreLÈs-NANCY, FRANCE 